Part $B$

Manuscript Number: JCOMB-D-16-00343R2

Title: A statistical treatment of the loss of stiffness during cyclic loading for short fiber reinforced injection molded composites

Article Type: Full length article

Keywords: C-Statistical properties/methods

B-Fatigue

E-Injection moulding

D-Mechanical testing

Corresponding Author: Dr. Atul JAIN,

Corresponding Author's Institution: KULeuven

First Author: Atul JAIN

Order of Authors: Atul JAIN; Wim Van Paepegem, PhD; Ignaas Verpoest, PhD; Stepan Lomov, PhD

Abstract: Injection molded short fiber reinforced composites (SFRC) have different local fiber orientation distribution (FOD) at every point. SN curves of short fiber reinforced composites are known to depend on the fiber orientation distribution. Such materials also suffer from continuous loss of stiffness during cyclic loading. It is not known whether the loss of stiffness is different for SFRC with different FOD. A statistical analysis of the loss of stiffness curves is presented in this paper. Tension-tension fatigue experiments are performed and loss of stiffness is collected for every data point in the SN curve. A systematic method for comparing the loss of stiffness is developed. It is concluded that the difference in loss of stiffness curves for coupons of SFRC with different FOD is not statistically significant. 
Dear Editor,

We, hereby submit revised version of article "A statistical treatment of the loss of stiffness during cyclic loading for short fiber reinforced injection molded composites" by the authors Atul Jain, Wim Van Paepegem, Ignaas Verpoest, Stepan V. Lomov to journal Composites Part B: Engineering

In this paper, a new method to statistically compare the loss of stiffness curves is developed and sufficiently high number of experiments are performed to compare the loss of stiffness curves of short fiber reinforced composites. It is seen that the loss of stiffness curves are independent of the fiber orientation distribution in the short fiber reinforced composites.

All the comments by all the referee have been addressed and described in the "answer to reviewer document".

We declare that the content is not duplicated and not submitted to any other journal concurrently.

The Corresponding author is

Atul Jain

Department of Metallurgy and Materials Engineering,

KU Leuven, Kasteelpark Arenberg 44 B-3001 Leuven,

Belgium;

Ph. No. 001-323-745-6354

Email: atuljain@usc.edu

Kind regards,

Atul Jain, Wim Van Paepegem, Ignaas Verpoest, Stepan V. Lomov 
We thank the Referee for his continued interest in our work and for helpful comments that will greatly improve the manuscript and we have tried to do our best to respond to the points raised. The Referee has brought up some useful points and we appreciate the opportunity to clarify our research objectives and methods.

As indicated below we have checked all the comments provided by the Referees and have made necessary changes accordingly to their indications. At the end of reply, wherever possible, we have indicated in bold the page number in text where change has been made. New text in the paper has been typed in red.

\section{Point 1: Influence of specimen extraction position (pag. 4)}

Profiles of the orientation tensor components over the thickness form process simulation are reported. An indication of the positions chosen for the plots of Fig.3a and Fig. 3b would be helpful for the reader. However, experimental evidence of FOD over the thickness is missing. Further, the influence of extraction position on mechanical properties is still not directly addressed. In view of this facts, it should be stated in the paper that "the FOD is uniform over the plate" is actually an assumption (supported by simulation) and that "extraction of specimens from different positions on the plate is a possible source of the scatter of quasistatic as well as fatigue strength".

Ans: The clarification has been added as suggested (page 4). Figure 2 has been improved by including points showing where simulation data was extracted from.

\section{Point 2: Fiber orientation distribution (pag. 3 and 4)}

It is well known that plates present a skin-core morphology, e.g. a variation of the FO over the thickness. This is clearly evident in the microscopic pictures of both papers by De Monte [5] and by Vincent [6]. I suggest thus to add a statement that experimental investigation of FO to validate the results from process simulation would be required. Indeed, I do not believe that the actual orientation is so uniform as predicted by SigmaSoft.

Ans: The point has been added (page 4, 5)

\section{Point 3: Initial value of stiffness (pag. 5)}

Initial stiffness values from fatigue tests have been reported in Tab. 2. I am however surprised that standard deviations for $0^{\circ}$ and $90^{\circ}$ are not reported since only two values are available. More than two values are however reported for these orientations in the paper, e.g. in Fig. 13b. It should be thus specified in the text whether initial stiffness has been computed for each sample or whether the mean value has been used for all the samples. 
Ans: In table 2, the values indicated are the measured stiffness by quasi-static tests performed on samples which survived million cycles (Figure 8a, b and c). Figure 9b, 11b and 13b

indicate the measured loss of stiffness during cyclic loading using extensometer (described in section 3.1 and figures 4 and 5a, b) for various cycles to failure. This point has been clarified in the manuscript and also table caption for table 2 (caption table 2, page 18 and page 11).

The stiffness has been normalized with the stiffness measured for first cycle for that specimen (page 12). 


\title{
A statistical treatment of the loss of stiffness during cyclic loading for short fiber
}

\section{reinforced injection molded composites}

\author{
Atul Jain ${ }^{*}, 1,2,{ }^{\mp}$, Wim Van Paepegem ${ }^{3}$, Ignaas Verpoest ${ }^{2}$, Stepan V. Lomov ${ }^{2}$
}

1. Siemens Industry Software NV, Interleuvenlaan 68, B-3001 Leuven;

2. Department of Materials Engineering , KU Leuven, Belgium;

3. Department of Material Science and Engineering, Ghent University, Belgium

*corresponding author email: atuljain@usc.edu

${ }^{\mp}$ Current affiliation: M.C. Gill Composites Center, University of Southern California

\begin{abstract}
Injection molded short fiber reinforced composites (SFRC) have different local fiber orientation distribution (FOD) at every point. SN curves of short fiber reinforced composites are known to depend on the fiber orientation distribution. Such materials also suffer from continuous loss of stiffness during cyclic loading. It is not known whether the loss of stiffness is different for SFRC with different FOD.

A statistical analysis of the loss of stiffness curves is presented in this paper. Tension-tension fatigue experiments are performed and loss of stiffness is collected for every data point in the SN curve. A systematic method for comparing the loss of stiffness is developed. It is concluded that the difference in loss of stiffness curves for coupons of SFRC with different FOD is not statistically significant.
\end{abstract}

\section{Introduction}

Increasingly composites are being looked at as possible replacements for metals in automobiles. This is due to the rising awareness and regulations towards reducing $\mathrm{CO}_{2}$ emissions. Short fiber reinforced composites (SFRC) have reasonable specific properties and are easy to manufacture on a large scale which makes them cost effective. Thus, they are ideal candidates for deployment in various industries like automotive etc. Optimum deployment of SFRC necessities that the fatigue behavior must be completely understood and simulated.

SFRC composites are usually made by the injection molding process, this process leads to different fiber orientation distribution (FOD) at every point in the part. It is known and has been experimentally confirmed that the SN curve depends on the fiber orientation distribution (FOD) of the SFRC [1-4]. Like most other polymer composite materials, SFRC are also known to suffer loss of stiffness when subjected to cyclic loading. There is a large amount of experimental evidence which confirms the loss of stiffness during cyclic loading particularly tension-tension fatigue [2,5-9]. 
There is very little known about the dependence of the loss of stiffness to the FOD of SFRC material. Based load and fiber length distribution but was independent of the orientation of the coupons, but an statistical proof of the same was not provided. Klimkeit et al. [9] observed greater loss of stiffness in the coupons with fibers in the loading direction as opposed to coupons with fiber in the transverse to loading direction. It is hard to make qualitative judgements about loss of stiffness curves since like all fatigue based quantities there is some inherent scatter and uncertainty. Two coupons having the same fiber content, length and orientation distribution; when subject to the same cyclic load may have different number of cycles to failure. Even if the number of cycles to failure are the same (or similar), the loss of stiffness could follow a different trend. Thus a thorough statistical treatment of the loss of stiffness is necessary to relieve the confusion.

Statistical methods to access variance in fatigue data have been tried for metals and welds [10,11]. Proper use of statistics can provide insights about composite fatigue. For example, Marshall et al. [12] using simple student test based statistics were able to prove that the scatter in his fatigue data of glass fiber UD composites was due to change in failure mode.

Apart from helping gaining further insight about the fatigue mechanisms in SFRC and dependence on the FOD the study of loss of stiffness and its dependence on FOD could be important for many reasons, a couple of them are highlighted here.

Recently, a hybrid multi-scale approach to derive the local SN curves was developed by Jain et al. [13-15]. Using a combination of the Mori-Tanaka formulation [16] and an algorithm for treating fiber matrix debonding [17], this method predicts the local SN curve with only one SN curve as input. The approach was named the Master SN curve approach, in short the MSNC approach. A major assumption of this formulation was that the damage propagation and subsequent loss of stiffness is the same for SFRC with different FOD. A statistical treatment of the loss of stiffness will confirm whether or not this assumption is true as it is suggested by good predictions reported in [13].

Also, during a SN curve based component level (high cycle) fatigue simulation of SFRC, each element in the RVE has a known FOD which is calculated using manufacturing simulation tools. Each element is treated as different material whose stiffness is typically calculated by mean field homogenization techniques [18], for example the Mori-Tanaka method [19]. The SN curve of every element is calculated either by interpolation or by other mechanics based methods for example the Master SN curve approach. The local loads are 
calculated using FE software by applying suitable boundary conditions and the reduced lifetime is derived from the local loads and the local SN curves. Apart from reduced lifetime, the composites also suffer from loss of stiffness which leads to stress redistribution and must be accounted for during fatigue simulation. A typical method is to reduce the stiffness of the elements and rerun the FE calculations after certain number of cycles [20]. If the loss of stiffness is the same for all FOD, this will simplify the calculations significantly as the loss of stiffness curve can be experimentally derived for one coupon with a certain FOD and the same loss of stiffness relation can be used for all the RVE. If the loss of stiffness is different for SFRC with different FOD then either additional tests have to be performed and/or relation between the FOD and loss of stiffness curves must be developed.

A physical justification of the assumption is that for SFRCs with different FOD (and FLD) but same constituents (fiber and matrix), the extent of damage (which is quantified by a loss of stiffness) needed to propagate and cause failure after same number of cycles should be similar. The expected damage events happening at the micro-level are expected to be similar, provided the FOD of the material is sufficiently random.

In this paper, a sufficiently large number of fatigue experiments are performed on SFRC with different FOD, the loss of stiffness curves are experimentally derived for each data point and a systematic method for the comparison of loss of stiffness is developed. For the scope of this paper only tension-tension fatigue tests are considered, but the method developed in this paper can be used for comparison of loss of stiffness curves at different applied load ratios as well.

Section 2 of this paper describes the experiments; the data extraction and analysis is elaborated in section 3. The experimental results are presented in section 4 , the statistical study is presented in section 5 . The conclusions are summarized in section 6.

\section{Experiments}

Polybutylene terephthalate (PBT) reinforced with 0.5 weight fraction of glass fiber (equivalent volume fraction is 0.35 ) compound was injection molded to plates having dimensions $170 \times 170 \times 2 \mathrm{~mm}$. The thickness of the plates are chosen to be as thin in order to ensure uniform FOD (with negligible core layer) through the thickness of the coupon based on the advice of Vincent et al. [21]. Coupons were machined from the plates in three directions, inclined at angles $\varphi=0,45$ and 90 with respect to the prevailing flow direction 
(Figure 1). For the rest of the paper, they are referred to as 0, 45 and 90-degree coupon respectively. Three coupons were machined from each plate for the 0 and 90-degree coupon, while only one coupon per plate was machined for the 45-degree coupon.

The plate used for injection molding has been designed so that the variation of the orientation is negligible in the areas where the coupons were machined. Also, the coupons are machined in such a way that the gauge length region of the horizontal, vertical and 45-degree coupon are all from the same square region in the center of the plate. This ensures that the variation of FOD (if any) within three horizontal coupons machined from the same plate will be the same as the variation of the FOD in the gauge length of one vertical coupon or the 45 -degree coupon (Figure 2). Thus, we are confident that there could be only minimal variability in the static and fatigue properties of the three coupons machined from different locations in the same plate (owing to some small variations in FOD). Also, the variability in the properties (for three coupons from same plate) if any, is the same as inevitable variance in local stiffness across the gauge length of a single coupon. This slight variation of properties for different coupons (and different regions in the same coupon) could add to scatter in the static and fatigue properties. This strategy of deriving several coupons from a single plate has been previously reported in literature [4], [22], [23]. Overall, it must be kept in mind that the confirmation of similar FOD for coupons machined from same plates but different locations is actually an assumption which is supported by simulation and other indirect observations. A direct FOD measurement through experimental means has not been reported. Instead, manufacturing simulation has been performed. For the analysis presented in this paper, this was seen as sufficient. Exact values of the FOD are not needed, we simply want to study of variance of loss of stiffness for coupons with different FOD and not perform any specific analysis which requires the exact values of the FOD as input. Thus, confirmation that the 0,45 and 90-degree coupons had sufficiently different FOD was enough.

Manufacturing simulation was performed on the plates by the commercial software SIGMASOFT [24]. The simulations are based on the Advani-Folgar [25] equation. The simulation was performed with the following process parameter set (the same as the one used for the injection molding process):

Melt temperature: $290^{\circ} \mathrm{C}$

Mold Temperature: $85^{\circ} \mathrm{C}$

Holding pressure: 700 bar

Holding pressure time: $3.5 \mathrm{sec}$

Filling time: $0.86 \mathrm{sec}$ 
Cooling time: $53.5 \mathrm{sec}$

There were 10 elements through the thickness of the plate. This number is confirmed based on a sensitivity analysis. No improvement or change was seen in the predicted FOD if the mesh is refined. The predicted variation of the FOD through the thickness was studied in the regions where coupons were machined. Simulation confirms that the variation of FOD in the region is minimal (less than $1 \%$ difference). The variation of the FOD through the thickness in two regions of the plate from where the coupons were machined has been given in Figure 3a, b. The simulations suggested the absence of "skin-core" distribution of orientation, this finding is consistent with the reported FOD for thin plates by De Monte et al.[26] who reported no variation of both experimental and simulated FOD through the thickness for thin plates of $1 \mathrm{~mm}$ and Vincent et al. [21] who used $2 \mathrm{~mm}$ plates. Also, a qualitative inspection of the fractured samples confirms the absence of the skin-core effect. We however, do believe that the FOD will not be as uniform as the simulation suggests, there can be some minor variation between the different layers, which the simulation cannot capture.

Comparison of the FOD in the two region also confirm that there is very little difference in the FOD of two coupons machined from different regions.

The FOD is characterized by the second order orientation tensor. The thickness weighted average of the second order orientation tensor has been reproduced below.

$a_{i j}=\left[\begin{array}{ccc}0.81 & 0.018 & 0.137 \\ 0.018 & 0.11 & 0.004 \\ 0.137 & 0.004 & 0.079\end{array}\right]$

The second order tensor is defined in the co-ordinate system such that the axis of the 0-degree coupon is axis 1 of the second-order tensor. Physically this means that the 0-degree coupon has fibers which are predominantly aligned in the loading direction, while the 90-degree coupon has fibers in the transverse to loading direction.

The coupons are subject to tensile and fatigue tests on a hydraulic horizontal Schenck fatigue testing machine. The machine was equipped with a load cell of $10 \mathrm{kN}$. Tests were load controlled and a sinusoidal load function with constant amplitude was applied. The applied loads had an R-ratio of 0.1 (pure tensiontension loading). Applied stresses were calculated dividing the applied load by the specimen net area, while the criterion to end the fatigue tests was complete specimen separation into two or more parts or no breakage until $10^{6}$ cycles. Coupons which survived a million cycles were counted as runouts and not used during the 
calculation of the SN curve. The tests were performed at a load frequency of $10 \mathrm{~Hz}$; this was chosen such that the temperature measured on the specimen surface did not exceed the test temperature by more than about $5^{\circ} \mathrm{C}$. Temperature monitoring was operated by a film type $\mathrm{NiCr}-\mathrm{Ni}$ thermocouple, clamped on the central part of the specimen surface. It was noticed that the rise of temperature in the surface of the specimen increases as the frequency of the applied load was increased and was about $3.3^{\circ} \mathrm{C}$ at $10 \mathrm{~Hz}$; it was decided not to go for higher frequencies to avoid resonance frequency problems with the machine. An extensometer was mounted on the specimens during the cyclic loading to keep track of the loss of stiffness during cyclic loading.

First, we tried to carry out tests from same load level and noticed that there was a significant difference in number of cycles to failure (more than a decade) even if the load was the same. Thus, we decided to do a large number of tests at varying load so that there would be enough number of tests having same/similar number of cycles to failure to perform statistical analysis. Both the integrated FE-fatigue simulation and the Master SN curve approach assume that for same number of cycles, the number of cycles to failure are the same for SFRC with different FOD. No assumptions are made about the variation of the loss of stiffness for the same applied load or SN curve fit equation. Thus, the analysis presented in this paper simply required that $\mathrm{SN}$ data be divided into groups where the range of number of cycle to failure is as small as possible and there are enough points to perform statistical analysis.

Coupons which survived million cycles of loading were subject to quasi-static tensile loading till failure after the fatigue tests. The rate of loading for these tests was programmed to be $0.5 \mathrm{~mm} / \mathrm{min}$. These tests were done to compare the pre and post-fatigue tensile behavior of SFRC.

\section{Data extraction and ANOVA analysis}

\subsection{Data extraction and analysis}

The loss of stiffness is tracked during cyclic loading by recording the load and displacement in the extensometer (which is mounted on the coupon) for every cycle (or for cycles with predefined interval). The frequency of the stiffness recording is progressively increased as the cycle number gets higher. For the same test, the loss of stiffness is recorded every cycle for the first 20 cycles, and subsequently every 20 cycles for the first 500 cycles. If the coupon survives more than 500 cycle, the loss of stiffness is recorded every 500 cycles. Loss of stiffness is calculated for each fatigue cycle by correlating the stress and strain in a linear fit. 
The slope of the fitted line is taken to be the secant modulus of the test coupon for a particular cycle (Figure

4).

The fit of the load displacement data to a straight line is ascertained by checking the value of the residual of the regression fit, $\mathrm{R}^{2}$ [27]. Typically, the residual of the regression fit, $\mathrm{R}^{2}$ is very low in the initial few cycles and subsequently approaches a consistent value close to 1 . This is because during fatigue tests on servohydraulic machines, the nominal stress amplitude is typically achieved only after a certain number of cycles, depending on the control parameters of the machine. The initial value of stiffness is calculated based on the first cycle after the value of the residual of the regression fit, $\mathrm{R}^{2}$ is higher than 0.95 . Based on previous experience with the machine on different loads, we were able to ensure that the nominal stress amplitude was reached before 10 cycles for most samples with a few exceptions of about 20 cycles. To further facilitate the stabilization of load with minimum number of cycles, we used low frequency $(1 \mathrm{~Hz})$ for the first 20 cycles and increased the frequency to desired value of $10 \mathrm{~Hz}$ in two steps only after the load had stabilized completely. The counting of the number of cycles to failure is also started from the cycle after the residual of the regression fit, $\mathrm{R}^{2}$ is higher than 0.95 .

Once the residual of the regression fit, $\mathrm{R}^{2}$ approached 0.95 , it was seen that the residual of the regression fit, $\mathrm{R}^{2}$ was remained consistently close to 1 (higher than 0.95 ) indicating the stiffness values are quite reliable. Two representative examples of the variation of the residual of the regression fit, $\mathrm{R}^{2}$ is given in Figure $\mathbf{5}$. Stiffness for a particular cycle is used to plot the loss of stiffness curve only if the value of $R^{2}$ was higher than 0.95 . For most fatigue tests, there were very few cycles where the value of $R^{2}$ was lower than the 0.95 . A loss of stiffness curve can be generated by plotting the number of cycles and corresponding secant stiffness for a test.

Tests which had more than 5\% rejected cycles were rejected and no longer considered for the loss of stiffness analysis. A total of 77 fatigue tests were performed out of which 5 tests had to be rejected due to unreliable load-displacement data.

Once the loss of stiffness curves have been generated, the next step is to make a systematic comparison of the different loss of stiffness curves for the 0,45 and 90 -degree coupons. In this paper the loss of stiffness curves are compared using statistical methods. The first step towards this is to fit the curves in some equation so that the co-efficient can be derived and compared for each variant (coupon type). An example of a loss of stiffness as a function of number of cycles is presented in Figure 6. A linear fit leads to unrealistic 
prediction of normalized stiffness higher 1.0, a quadratic fit with intercept set to 1 is seen to yield a better fit to the experimental curves.

The loss of stiffness curve can be fitted to a number of equations namely linear in log-log plot or semi-log plot; quadratic with unit intercept in $\log$-log or semi log plot. Of the various possibilities it is seen that a quadratic with unit intercept in a log-log plot gave the best fit to the curve. This is quantitatively confirmed by the highest value of $\mathrm{R}^{2}$ for a quadratic fit in a log-log plot. Though the analysis presented in this paper considers loss of stiffness in a log-log plot, the same analysis can also be repeated with same conclusions if a semi-log plot is considered. Similar analysis considering linear fit has been previously presented for only high cycle fatigue by the same authors in a conference paper [14]. In this paper we have used a quadratic fit because of the higher residual of regression values and due to the fact that the non-physical intercept of greater than one has been avoided. A summary of the values of the residual of the regression fit $\mathrm{R}^{2}$ showing better fit for quadratic equation has been presented in Table 1.

Mathematically the quadratic fit equation is as follows:

$\log \left(E_{N}\right)=\operatorname{coeff} 1 \times \log N \times \log N+\operatorname{coeff} 2 \times \log N$

In this equation coeff 1 and coeff 2 are the two constants. Thus, each loss of stiffness curve is characterized by two constants which will be compared by statistical tests.

\subsection{Comparison of loss of stiffness curves}

There are a number of ways to compare three or more sets of data and ascertain the significance of difference in the mean values of the different groups. Probably the most common among them is the analysis of variance (ANOVA). However, the ANOVA analysis has two important assumptions that must be fulfilled before reliable use.

- The population is normally distributed

- Homogeneity of variances

For small sets of data (sample size less than 4), there are no reliable ways to ascertain a normal distribution of population. In such cases, a non-parametric version of ANOVA is often used. For this paper, we propose to use the Kruskal Wallis test [28], this method is also commonly known as "one-way ANOVA on ranks". This method is known to be the most reliable method for testing samples where the sample sizes are unequal 
and the either of the two above assumptions are violated. However, this method has the known drawback that it is less robust if used in cases when the assumptions of ANOVA are valid.

In this method, the p-value of the Kruskal Wallis test on the data is calculated and compared with the value of alpha (or the chosen significance level). The hypothesis of similarity of data is supported if the p-value is higher than the value of alpha. First, the chi-square value is calculated which is approximated by following equation

$H=\frac{12}{n(n+1)} \sum_{j=1}^{k} \frac{R_{j}^{2}}{n_{j}}-3(n+1)$

Where, $k=$ the number of groups, $n_{j}$ is the size of the $j^{\text {th }}$ group, $R_{j}$ is the rank sum for the $j^{\text {th }}$ group and $n$ is the total sample size. The p-value of the test is subsequently calculated as the right tailed probability of the chi-squared distribution calculated in equation 2.

First, the measured loss of stiffness before final failure is compared. The significance level of $95 \%$ is used for this comparison. Next, the loss of stiffness curves are compared. For the comparison of the curves, first the curves are fit into equations (which have been described in section 3.1) and the values of the coefficients are compared.

Three steps are performed for a statistical comparison of the curves

i. The measured loss of stiffness data are fit to the quadratic equation and the residual of the regression fit $\left(\mathrm{R}^{2}\right)$ is compared to ensure that the curves fit similarly to the power equation. This is a necessary step before Kruskal Wallis test can be performed.

If there is a large difference in the values of the residual of the regression fit for different tests, then the Kruskal Wallis test can no longer be applied reliably.

ii. The first constant of the curve (coeff. 1 from equation 1) is compared and Kruskal Wallis test is performed. For the calculations a $95 \%$ significance is tested.

iii. The second constants are also compared in a similar manner for a significance level of 95\%

Therefore, the hypothesis of the similarity of the different loss of stiffness curves is tested for a composite hypothesis of $90.25 \%(=95 \times 95 \%)$ significance. $90 \%$ confidence interval is admittedly not a very stringent criterion; this criterion was chosen since it is common practice to perform design analysis based on $90 \%$ 
confidence intervals. A number of attempts at fatigue design analysis using confidence intervals of $90 \%$ have been reported in literature for a wide range of materials including composites and metals [11, 22-28]. Apart from the loss of stiffness curves, the similarity of the measured loss stiffness just before final failure is also compared.

The data analysis for this paper was generated using the Real Statistics Resource Pack software [36] addin in Microsoft excel.

\section{Results}

The SN curve derived by experiments are given in Figure 7. It should be noted that the SN curve has been previously reported [13]. The value of $\mathrm{T}_{\sigma}$ has been calculated by first calculating the $90 \%$ confidence interval and then subsequently determining the ratio of the upper and the lower limits as prescribed by De Monte et al.[2]. The value of $\mathrm{T}_{\sigma}$ is found to be varying in between 1.24 and 1.28, these numbers are higher than expected but not completely unusual for SFRC. For example, De Monte et al. who reported range of 1.14-1.23 for coupons subjected to tension-tension loading and in between 1.05-1.30 for loading at different r-ratio. By carefully extracting the published fatigue data and calculating the value of $\mathrm{T}_{\sigma}$ in the above described way, one sees that the value of $\mathrm{T}_{\sigma}$ for the data by Klimkeit et al. [6] comes to be in the range of 1.22-1.35; while that from Mortazavian and Fatemi [4] are in the range of 1.18-1.36.

In this paper, the loss of stiffness has been compared. Depending on the number of cycles to failure, the fatigue tests were divided in three groups as shown in Figure 7. The rationale behind choosing similar number of cycles to failure to group the SN data is that we intended to compare the loss of stiffness for tests with similar number of cycles to failure and not the same load. Both the integrated FE-fatigue simulation and the Master SN curve approach assume that for same number of cycles, the number of cycles to failure are the same for SFRC with different FOD. No assumptions are made about the variation of the loss of stiffness for the same applied load or SN curve fit equation.

Another possibility to group the SN data could be on the basis of same/similar load as a function of the ultimate tensile strength (UTS). However, if the SN data is grouped on the basis on same/similar loads fraction, there are no direct ways to use the scatter in the loss of stiffness for comparing the loss of stiffness curves across different FOD. We have already shown that the SN curves for different FOD do not have the same UTS proportionality [13]. 
The groups are chosen in such a way that there are at least 3 points per group for every coupon type. The performing the MSNC algorithm at three points $\left(10^{4}, 10^{5}\right.$ and $\left.10^{6}\right)$ cycles and we wanted to test the assumptions of the MSNC approach for each of the three ranges. The range of number of cycles to failure for the three groups is as follows:

High cycle region (group 1): $10^{6}$ Cycles

Mid cycle region (group 2): 279940 to 20673 cycles

Low cycle region (group 3): 8072 to 1261 cycles

For each of the three groups, ANOVA analysis is performed and the loss of stiffness curves are compared as described in section 3. It is important to note at this point the fact that while groups 2 and 3 consist of coupons which have failed. Thus all the coupons in this group have same state to damage at the end of tests. However, this is not the case for the samples belonging to group 1 (runouts). The statistical analysis for group 1 is presented for the sake of completeness with the aim of comparting the loss of stiffness across the entire range of thousand to million cycles. This comparison is based on the assumption that the eventual number of cycles to failure of the runout coupons are within the same (similar) range as the other two groups: group 2 (13.5) and group 3 (6.4). Looking at the SN data (Figure 7), this seems to be a reasonable assumption. However, there is no way to validate this assumption, the statistical results of the group 1 must be interpreted with this disclaimer in mind.

The stress-strain curves for virgin and the fatigued samples are shown in Figure 8 for 0, 45 and 90-degree coupon respectively. It was not possible to perform the tensile tests on each of the coupons which survived million cycles due to problems while clamping the coupons in the tensile test machine. It was seen that the coupons broke in the clamps when the grips were tightened. There is probably some damage and fatigue introduced in the shoulder of the dog bone samples during the fatigue testing in the horizontal Schenck. A summary of the stiffness, UTS and strain to failure for the 0,45 and 90 -degree coupons measured by quasistatic testing is presented in Table 2.

It is seen that there is a definite loss of stiffness and strength for the fatigued coupons. However, the strain to failure was similar for both the virgin and fatigued coupons. There is a large amount of scatter in the UTS as well as strain to failure (for both the virgin and fatigued samples), this is consistent with the somewhat high 
scatter also observed in the fatigue tests. Further by calculating the $90 \%$ confidence intervals of the UTS distribution and calculating the $\mathrm{T}_{\sigma}$ (ratio of upper and lower limit of $90 \%$ confidence interval), one sees that the value of the $\mathrm{T}_{\sigma}$ is about $1.22,1.1$ and 1.1 for the 0,45 and 90-degree coupon respectively. As evident from the plots the values are somewhat high but this is lower than the value of fatigue scatter of 1.25 to 1.28 (which is also expected since fatigue properties tend to have more scatter than the quasi-static properties). This somewhat higher scatter is probably due to the high fiber content. High fiber content in SFRC is expected to cause greater scatter in strength properties due to greater probability of fiber clusters and stress concentrations.

Also, it is worth noting that the initial stiffness measured by the fatigue tests match well with the quasi-static tests. The average stiffness measured by the quasi-static tests were $17.06,9.15$ and $8.35 \mathrm{GPa}$ respectively for the 0,45 and 90-degree coupon, while the average initial stiffness measured by the fatigue tests were 17.3, 9.7 and 8.7 GPa respectively.

\title{
4.1 Group 1
}

In this subsection the loss of stiffness data for the group 1 is presented and fit to quadratic fit. In each of the three subsequent sections, the stiffness has been normalized with the stiffness measured for first cycle for that specimen.

\subsubsection{Measured loss of stiffness}

The loss of stiffness curves for the 0, 45 and 90-degree coupons which survived a million cycles is presented in Figure 9a. A qualitative analysis of the plot confirms that the loss of stiffness during cyclic loading is independent of the FOD of the coupon; this will be confirmed by Kruskal Wallis test (in section 5). The normalized stiffness measured in the final cycle of loading of the coupons is shown in Figure 9b. The average loss of stiffness for the 0,45 and 90 -degree coupon was measured to be $9.7 \pm 1.1,10.3 \pm 1.5$ and $10.1 \pm 2.4 \%$ respectively.

\subsubsection{Quadratic fit with unit intercept}

The curves are fit to a quadratic equation and the histogram of the two coefficients are presented in Figure10a, b. It is seen that the value of the coefficient 2 is consistently close to 0 .

\author{
4.2 Group 2 \\ 4.2.1 Measured loss of stiffness
}


The loss of stiffness curves for the 0, 45 and 90-degree coupons which survived between 279940 and 20673

cycles is presented in Figure 11a. The normalized stiffness measured in the final cycle of loading of the coupons is shown in Figure 11b. The average loss of stiffness for the 0, 45 and 90-degree coupon was measured to be $13.0 \pm 3.2,13.6 \pm 1.03$ and $12.3 \pm 5.7 \%$ respectively.

\subsubsection{Quadratic fit with unit intercept}

Similar to group 1 , the value of the co-eff 2 is seen to be consistently close to zero and the value of $\mathrm{R}^{2}$ is seen to be higher than the previous group with minimum value equal to 0.93 . The histogram of co-eff 1 and co-eff 2 are shown in Figure 12a, b.

\subsection{Group 3}

4.3.1 Measured loss of stiffness

The loss of stiffness curves of fatigue tests lying in group 3 are presented in Figure 13a. The normalized stiffness measured in the final cycle of loading of the coupons is shown in Figure 13b. The average loss of stiffness for the 0, 45 and 90-degree coupon was measured to be $16.8 \pm 2.8,15.6 \pm 2.8$ and $13.5 \pm 1.7 \%$ respectively.

\subsubsection{Quadratic fit with zero intercept}

The histogram of co-eff 1 and co-eff 2 for a quadratic with zero intercept is shown in Figure 14a, b.

\section{Statistical analysis}

For each of the three groups, p-value corresponding to the data set is calculated and compared with the value of allowed significance level to ascertain the hypothesis of similarity of the curves. The p-values of the three groups are summarized in Table 3. It was observed that the p-value is higher than 0.05 in all the cases thus confirming that the loss of stiffness curves for different orientation but similar number of cycles to failure are statistically similar.

A direct comparison of the similarity of the loss of stiffness curves across different groups is not possible since the end state of damage in group 1 is not the same as groups 2 and 3.

This conclusion of the statistical insignificance in the loss of stiffness curve is despite some observable trends in the intercept and negative slope histograms. For example (in Figure 13b), the value of the measured loss of stiffness seems to be increasing from 0-degree to 90-degree. These two contrasting 
observations can be explained by reasoning that the conditions ascribed for statistical comparisons (90\% confidence interval) are not very stringent. Hence the conclusion on the statistical similarity is valid within the given confidence intervals. If more restrictive requirements are presented, then more detailed analysis of the trends (involving more voluminous experimental program) will be needed.

\section{Conclusions}

A method to statistically compare the loss of stiffness curves of composites has been developed. Fatigue experiments were performed and the loss of stiffness curves were collected.

For similar number of cycles, the loss of stiffness just before failure is confirmed to be the same for SFRC coupons with different FOD. Also it is confirmed with a confidence of $90 \%$ that the difference in the loss of stiffness curves is not statistically significant. The assumptions of the Master SN curve stand validated. During the finite element rerun during fatigue simulation of SFRC, same loss of stiffness expression can be used for the different RVE even though they may have different FOD. This idea of treating loss of stiffness as the same for different RVE has been implemented in LMS Virtual.Lab Durability [37] part of Siemens Industry Software [38].

There is a very slight dependency on the number of cycles, the average loss of stiffness in the high cycle region was slightly less than low cycle region.

In this paper, we intended to compare the loss of stiffness for tests with similar number of cycles to failure. Quantifying the loss of stiffness dependence on the applied load and different applied load-ratio are interesting problems and will be looked at in future work.

\section{Acknowledgement}

The authors wish to thank the IWT Vlaanderen for funding this research as a part of the project "Fatigue life prediction of random fiber composites using hybrid multi-scale modelling methods" - COMPFAT Baekeland mandate number 100689. Thanks also to Stefan Straesser, Christophe Liefooghe and Dr. Michael Hack from Siemens PLM for useful discussions and constructive feedback. I. Verpoest and S.V. Lomov are holders of the Toray Chair in Composites at KULeuven.

\section{References}


short glass fibre reinforced polyamide-6. Int J Fatigue 2007;29:199-208. doi:http://dx.doi.org/10.1016/j.ijfatigue.2006.04.001.

[2] De Monte M, Moosbrugger E, Quaresimin M. Influence of temperature and thickness on the off-axis behaviour of short glass fibre reinforced polyamide 6.6 cyclic loading. Compos Part A Appl Sci Manuf 2012;41:1368-79. doi:http://dx.doi.org/10.1016/j.compositesa.2010.02.004.

[3] Mortazavian S, Fatemi A. Fatigue behavior and modeling of short fiber reinforced polymer composites: A literature review. Int J Fatigue 2015;70:297-321. doi:10.1016/j.ijfatigue.2014.10.005.

[4] Mortazavian S, Fatemi A. Fatigue behavior and modeling of short fiber reinforced polymer composites including anisotropy and temperature effects. Int J Fatigue 2015;77:12-27. doi:10.1016/j.ijfatigue.2015.02.020.

[5] Arif MF, Saintier N, Meraghni F, Fitoussi J, Chemisky Y, Robert G. Multiscale fatigue damage characterization in short glass fiber reinforced polyamide-66. Compos Part B-Engineering 2014;61:55-65. doi:10.1016/j.compositesb.2014.01.019.

[6] Klimkeit B, Nadot Y, Castagnet S, Nadot-Martin C, Dumas C, Bergamo S, et al. Multiaxial fatigue life assessment for reinforced polymers. Int J Fatigue 2011;33:766-80. doi:10.1016/j.ijfatigue.2010.12.004.

[7] Arif MF, Meraghni F, Chemisky Y, Despringre N, Robert G. In situ damage mechanisms investigation of PA66/GF30 composite: Effect of relative humidity. Compos Part B-Engineering 2014;58:487-95. doi:10.1016/j.compositesb.2013.11.001.

[8] Mallick PK, Zhou Y. Effect of mean stress on the stress-controlled fatigue of a short E-glass fiber reinforced polyamide-6,6. Int J 2004;26:941-6. doi:http://dx.doi.org/10.1016/j.ijfatigue.2004.02.003.

[9] Klimkeit B, Castagnet S, Nadot Y, Habib A El, Benoit G, Bergamo S, et al. Fatigue damage mechanisms in short fiber reinforced PBT+PET GF30. Mater Sci Eng A 2011;528:1577-88. doi:http://dx.doi.org/10.1016/j.msea.2010.10.081.

[10] Schneider CRA, Maddox SJ. Best practice guide on statistical analysis of fatigue data. Weld Inst Stat Rep 2003.

[11] Sutherland HJ, Veers PS. The development of confidence limits for fatigue strength data. 2000 ASME Wind Energy Symp., 2000, p. 413-23.

[12] Marshall IH, Barnard PM, Butler RJ, Curtis PT. Fatigue Scatter of UD Glass Epoxy, a Fact or Fiction? Compos. Struct. 3, Springer Netherlands; 1985, p. 69-82. doi:10.1007/978-94-009-4952-2_5.

[13] Jain A, Beas J, Van Paepegem W, I. V, Lomov S. The Master SN curve approach - A hybrid multiscale approach towards fatigue simulation of short fiber reinforced composites. Compos Part A Appl Sci Manuf 2015;Accepted. doi:doi:10.1016/j.compositesa.2015.11.038.

[14] Jain A, Abdin Y, Straesser S, Van Paepegem W, Verpoest I, Lomov S V. MASTER SN CURVE APPROACH-A HYBRID MULTISCALE APPROACH TO FATIGUE SIMULATION OF SHORT FIBER COMPOSITES. 20th Int. Conf. Compos. Mater., Copenhagen: 2015.

[15] Jain A, Van Paepegem W, Verpoest I, Lomov S V. A feasibility study of the Master SN curve approach for short fiber reinforced composites. Int J Fatigue 2016.

[16] Jain A, Lomov S V., Abdin Y, Verpoest I, Van Paepegem W. Pseudo-grain discretization and full Mori Tanaka formulation for random heterogeneous media: Predictive abilities for stresses in individual 

doi:10.1016/j.compscitech.2013.08.009.

[17] Jain A, Abdin Y, Van Paepegem W, Verpoest I, Lomov S V. Effective anisotropic stiffness of inclusions with debonded interface for Eshelby-based models. Compos Struct 2015;131:692-706. doi:10.1016/j.compstruct.2015.06.007.

[18] Jain A, Verpoest I, Hack M, Lomov S, Adam L, Van Paepegem W. Fatigue Life Simulation on Fiber Reinforced Composites - Overview and Methods of Analysis for the Automotive Industry. SAE Int J Mater Manuf 2012;5:205-14. doi:10.4271/2012-01-0730.

[19] Mori T, Tanaka K. Average Stress in Matrix and Average Elastic Energy of Materials with Misfitting Inclusions. Acta Metall 1973;21:571-4.

[20] Van Paepegem W, Degrieck J, De Baets P. Finite element approach for modelling fatigue damage in fibre-reinforced composite materials. Compos Part B-Engineering 2001;32:575-88. doi:10.1016/s1359-8368(01)00038-5.

[21] Vincent M, Giroud T, Clarke A, Eberhardt C. Description and modeling of fiber orientation in injection molding of fiber reinforced thermoplastics. Polymer (Guildf) 2005;46:6719-25. doi:http://dx.doi.org/10.1016/j.polymer.2005.05.026.

[22] BERNASCONI A, DAVOLI P, BASILE A, FILIPPI A. Effect of fibre orientation on the fatigue behaviour of a short glass fibre reinforced polyamide-6. Int J Fatigue 2007;29:199-208. doi:10.1016/j.ijfatigue.2006.04.001.

[23] Zhou YX, Mallick PK. Fatigue performance of an injection-molded short E-glass fiber-reinforced polyamide 6,6. I. Effects of orientation, holes, and weld line. Polym Compos 2006;27:230-7. doi:10.1002/pc.20182.

[24] Sigmasoft. SIGMA Engineering GmbH, Aachen. Http://www.sigmasoft.de/ 2014. doi:http://www.sigmasoft.de/.

[25] Folgar F, Tucker CL. Orientation behavior of fibers in concentrated suspensions. J Reinf Plast Compos 1984;3:98-119.

[26] De Monte M, Moosbrugger E, Quaresimin M. Influence of temperature and thickness on the off-axis behaviour of short glass fibre reinforced polyamide 6.6-Quasi-static loading. Compos Part a-Applied Sci Manuf 2010;41:1368-79. doi:http://dx.doi.org/10.1016/j.compositesa.2010.02.004.

[27] Draper NR, Smith H. Applied regression analysis. John Wiley \& Sons; 2014.

[28] Kruskal WH, Wallis WA. Use of Ranks in One-Criterion Variance Analysis. J Am Stat Assoc 1952;47:583-621. doi:10.1080/01621459.1952.10483441.

[29] Ren XD, Zhang YK, Jiang DW, Zhang T, Sun GF. A model for reliability and confidence level in fatigue statistical calculation. Theor Appl Fract Mech 2012;59:29-33. doi:10.1016/j.tafmec.2012.05.004.

[30] Lassen T, Darcis P, Recho N. Fatigue Behavior of Welded Joints Part 1-Statistical Methods for Fatigue Life Prediction. Weld J 2005;84:183-7.

[31] Gope P. Scatter Analysis of Fatigue Life and Prediction of S-N Curve. J Fail Anal Prev 2012;12:507-17. doi:10.1007/s11668-012-9590-0.

[32] Chen Z, Zheng S. Lifetime distribution based degradation analysis. Reliab IEEE Trans 2005;54:3-10. 
[33] Murthy PLN, Mital SK, Gyekenyesi JZ, Gyekenyesi JP. Reliability and Creep/Fatigue Analysis of a CMC Component. Cleveland, Ohio 44135: 2007.

[34] Wang X, Sun JQ. On the fatigue analysis of non-Gaussian stress processes with asymmetric distribution. J Vib Acoust 2005;127:556-65.

[35] Reigosa PD, Wang H, Yang Y, Blaabjerg F. Prediction of bond wire fatigue of IGBTs in a PV inverter under long-term operation. Appl Power Electron Conf Expo (APEC), 2015 IEEE 2015:3052-9. doi:10.1109/APEC.2015.7104787.

[36] Zaiontz C. Real Statistics Using Excel 2016.

[37] http://www.plm.automation.siemens.com/en_us/products/Ims/virtual-lab/durability/, editor. LMS Virtual.Lab Durability-Durability analysis for optimal product performance 2015. doi:http://www.plm.automation.siemens.com/en_us/products/Ims/virtual-lab/durability/.

[38] www.siemens.com/plm, editor. Siemens PLM software. Www.siemens.com/plm 2015. doi:www.siemens.com/plm.

Figure 1 Specimens preparation (a) plates used for injection molding. (b)The geometry of the dog bone specimen with dimensions in $\mathrm{mm}$; (c) orientation designation of coupon.

Figure 2 A representation of the plate showing regions of the three horizontal coupon and 45-degree coupon being machined showing that the variation in the FOD between 3 coupons can be expected to be the same as inter-coupon variation for the 45-degree coupon. The dimensions of the plate and the coupons are drawn to scale. Markers indicate the point whose variation of predicted FOD is shown in Figure 3. Interior dotted lines indicate region where manufacturing simulation was studied.

Figure 3 Variation of FOD as predicted by the manufacturing simulation software, the elements are extracted through the thickness of the plate. (a) center of the plate (center of the horizontal coupon 2 in figure 2) (b) right side (center of the horizontal coupon 1 in figure 2)

Figure 4 Typical stress-strain displacement curve during cyclic loading for 0-degree coupon. A linear relationship is observed and the slope of the line is the stiffness of the material.

Figure 5 Variation of the residual of the regression fit parameter, $\mathrm{R}^{2}$ for linear fit of stress-strain data. Dotted lines indicate the minimum allowed value of the regression fit parameter, $\mathrm{R}^{2}$ for a reliable value of the stiffness calculations a) 45-degree coupon, maximum applied load was $32 \mathrm{MPa}$. b) 0-degree coupon, maximum applied load was 55MPa

Figure 6 Typical loss of stiffness curves in log-log plot for a 90-degree coupon, applied load is $30 \mathrm{MPa}$ and the number of cycles to failure is 117280 cycles. The loss of stiffness is fitted to linear and quadratic fits. 
Figure $7 \mathrm{SN}$ curve of $50 \%$ glass fiber reinforced PBT, coupons are machined from injection molded plates confidence interval limits based on a linear fit in log-log plot.

Figure 8 Stress-strain behavior of coupons which survived a million cycles and virgin coupons. (a) 0-degree coupon, (b) 45-degree coupon and (c) 90-degree coupon

Figure 9 Loss of stiffness measurements (a) Loss of stiffness curves for 0, 45 and 90-degree coupons which survived a million cycles (b) Measured normalized stiffness before the final cycle of loading

Figure 10 Quadratic curve fit parameters for 0, 45 and 90-degree coupons which survived a million cycles. (a) Coeff-1, the value above each bar indicates the value of $\mathrm{R}^{2}$ for the particular curve (b) Coeff-2, the $\mathrm{y}$-axis in this plot is logarithmic to show the low values

Figure 11 Loss of stiffness curves measurements for 0, 45 and 90-degree coupons which survived between 279940 and 20673 cycles before failure. (a) Loss of stiffness curves, both the axes are logarithmic (b) Histogram of normalized measured stiffness before final failure, number over each bar indicates the number of cycles before failure

Figure 2 Quadratic curve fit parameters for 0, 45 and 90-degree coupons which survived between 279940 and 20673 cycles before failure. (a) Coeff-1, the value above each bar indicates the value of $\mathrm{R}^{2}$ for the particular curve (b) Coeff-2, the y-axis in this plot is logarithmic to show the low values

Figure 3 Loss of stiffness curves comparisons for 0, 45 and 90-degree coupons which survived between 8072 and 1261 cycles before failure. (a) Loss of stiffness curves, both the axes are logarithmic (b) Histogram of normalized measured stiffness before final failure, number over each bar indicates the number of cycles before failure

Figure 4 Quadratic curve fit parameters for 0, 45 and 90-degree coupons which survived between 8072 and 1261 cycles before failure. (a) Coeff- 1 , the value above each bar indicates the value of $\mathrm{R}^{2}$ for the particular curve (b) Coeff-2, the y-axis in this plot is logarithmic to show the low values

Table 1 Comparison of the values of the residual of the regression fit for linear and quadratic fit. 
Table 2 A comparison of the stiffness, UTS and modulus of the virgin and fatigued samples for 0, 45 and

90-degree coupon measured by quasi-static testing. The standard deviation for the fatigued 0 and 45 -degree sample is not presented since the number of datasets is only 2 .

Table 3 The p-value of the Kruskal Wallis test for the measured loss of stiffness and curve fit parameter for quadratic fit for the three groups, a value higher than 0.05 indicates that the difference between the groups is not significant. 
Table 1 Comparison of the values of the residual of the regression fit for linear and quadratic fit.

\begin{tabular}{|c|c|c|}
\hline Test & $\begin{array}{l}\text { Linear } \\
\text { fit }\end{array}$ & $\begin{array}{l}\text { Quadratic } \\
\text { fit }\end{array}$ \\
\hline \multirow{4}{*}{ 0-degree } & 0.85 & 0.86 \\
\hline & 0.90 & 0.92 \\
\hline & 0.89 & 0.88 \\
\hline & 0.90 & 0.90 \\
\hline \multirow{4}{*}{ 45-degree } & 0.90 & 0.90 \\
\hline & 0.95 & 0.94 \\
\hline & 0.83 & 0.87 \\
\hline & 0.83 & 0.94 \\
\hline \multirow{3}{*}{ 90-degree } & 0.86 & 0.87 \\
\hline & 0.82 & 0.90 \\
\hline & 0.84 & 0.98 \\
\hline
\end{tabular}

Table 2 A comparison of the stiffness, UTS and modulus of the virgin and fatigued samples for 0,45 and 90 degree coupon. The standard deviation for the fatigued 0 and 90 degree sample is not presented since the number of datasets is only 2 .

\begin{tabular}{|c|l|c|c|c|}
\hline & & 0 -degree & 45 -degree & 90 -degree \\
\hline \multirow{3}{*}{$\begin{array}{c}\text { Virgin } \\
\text { sample }\end{array}$} & Stiffness, in GPa & $17.06 \pm 0.47$ & $9.15 \pm 0.75$ & $8.35 \pm 0.465$ \\
\cline { 2 - 5 } & UTS, in MPa & $126 \pm 15$ & $65 \pm 4.8$ & $44 \pm 2.8$ \\
\cline { 2 - 5 } & Strain to failure, \% & $0.97 \pm 0.23$ & $1.08 \pm 0.21$ & $0.63 \pm 0.8$ \\
\hline \multirow{2}{*}{$\begin{array}{c}\text { Fatigued } \\
\text { sample }\end{array}$} & Stiffness, in GPa & $14.9 \pm-$ & $8.2 \pm 0.38$ & $7.45 \pm-$ \\
\cline { 2 - 5 } & UTS, in MPa & $70.47 \pm-$ & $59.22 \pm 3.04$ & $38.2 \pm-$ \\
\cline { 2 - 5 } & Strain to failure, \% & $0.57 \pm-$ & $1.19 \pm 0.08$ & $0.66 \pm-$ \\
\hline
\end{tabular}

Table 3 The $p$-value of the Kruskal Wallis test for the measured loss of stiffness and curve fit parameter for quadratic fit for the three groups, a value higher than 0.05 indicates that the difference between the groups is not significant.

\begin{tabular}{|l|r|r|r|}
\hline & \multicolumn{1}{|l|}{$\begin{array}{l}\text { loss of } \\
\text { stiffness }\end{array}$} & co-eff. 1 & co-eff. 2 \\
\hline high cycle & 0.70 & 0.46 & 0.42 \\
\hline mid cycle & 0.98 & 0.63 & 0.42 \\
\hline low cycle & 0.16 & 0.65 & 0.98 \\
\hline
\end{tabular}




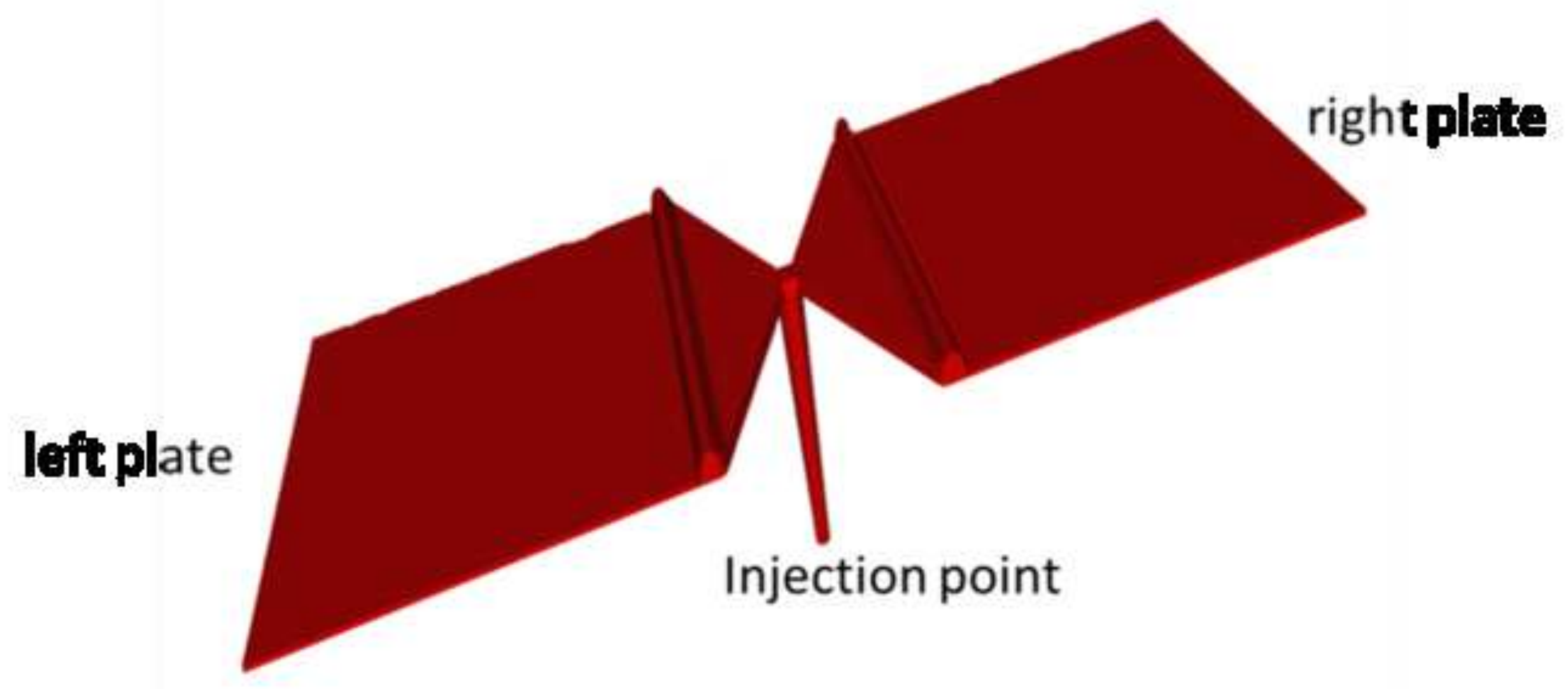


Click here to download high resolution image

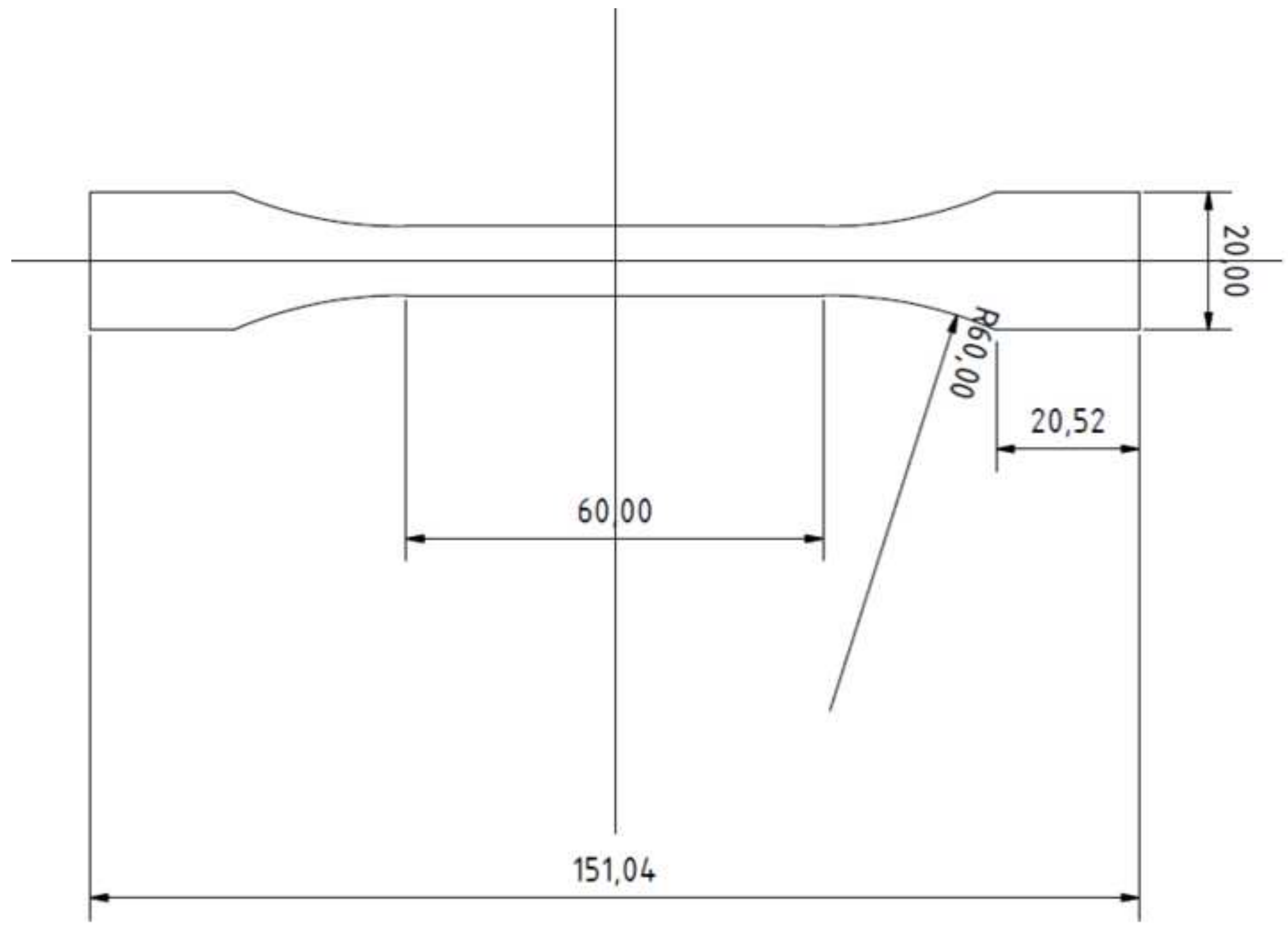


Figure 1c
Click here to download high resolution image

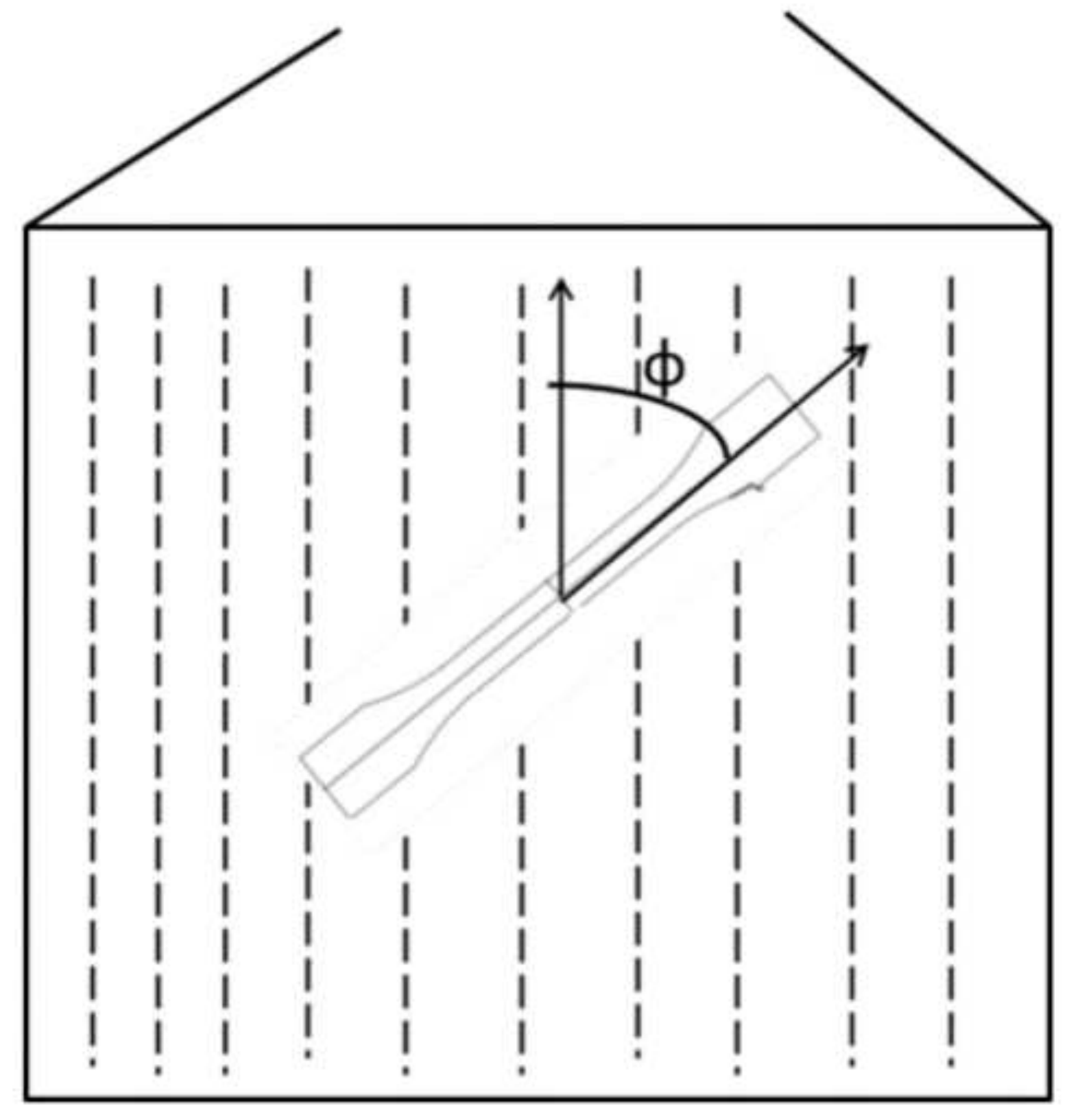




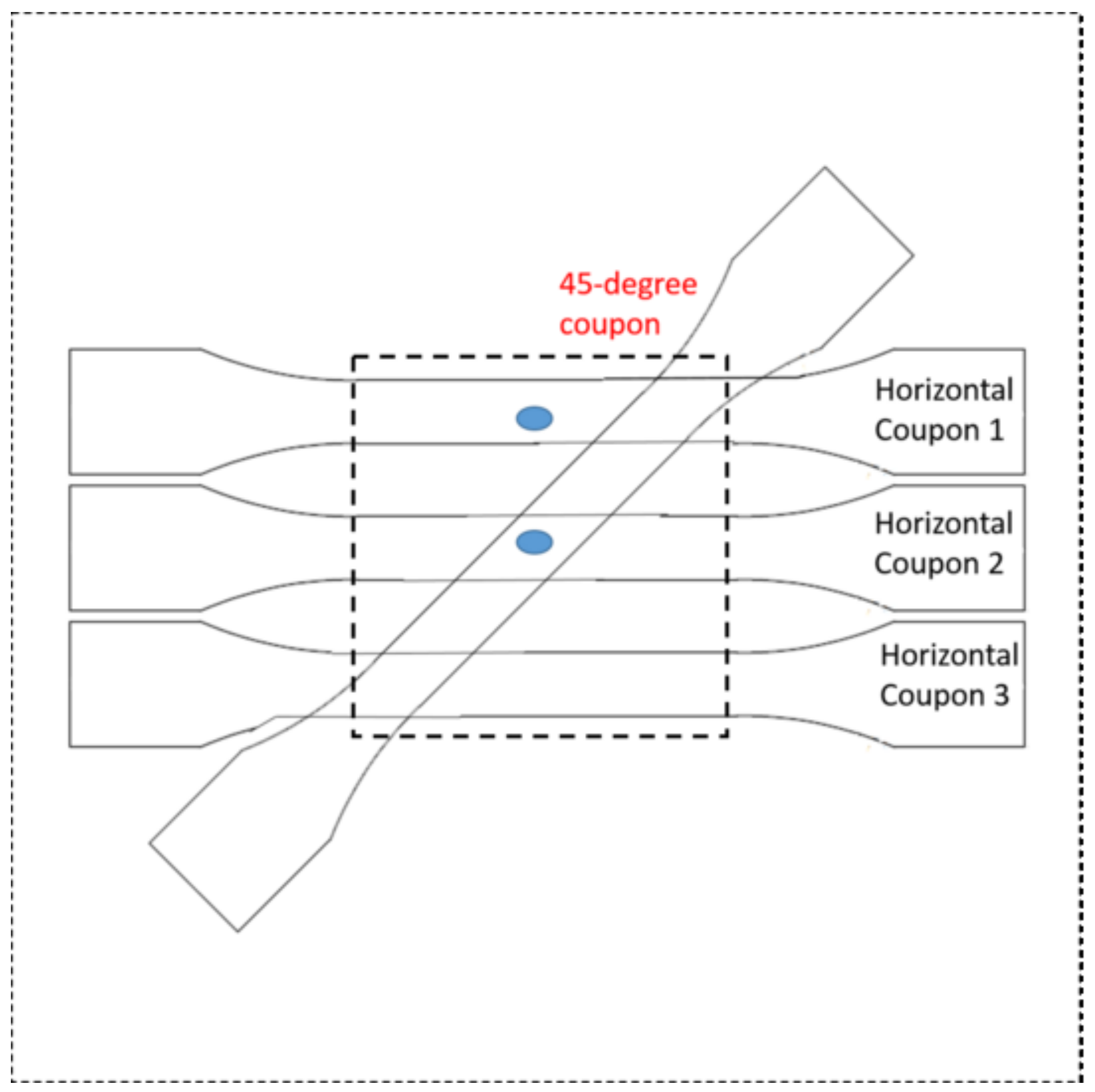


Click here to download high resolution image

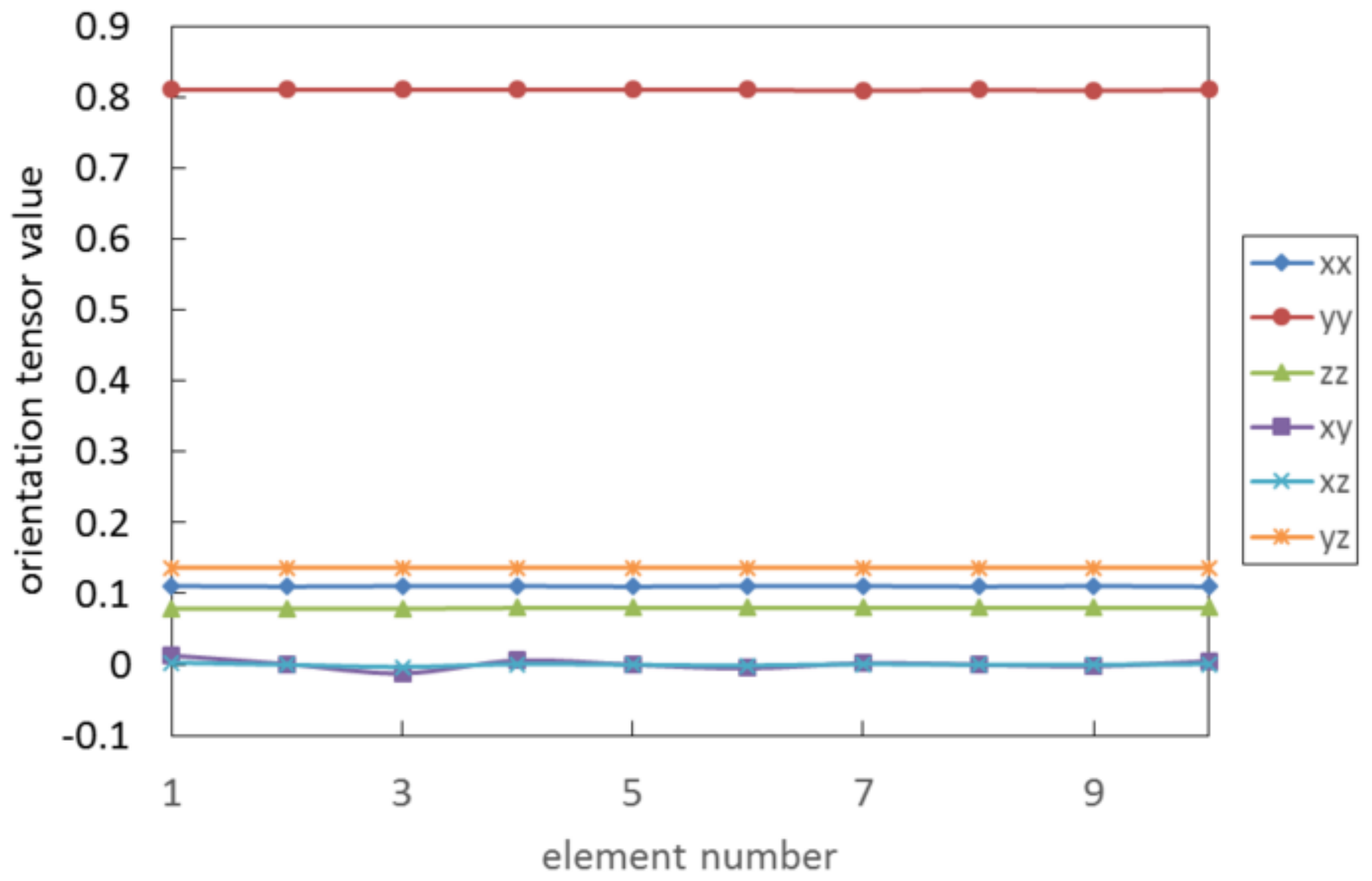


Click here to download high resolution image

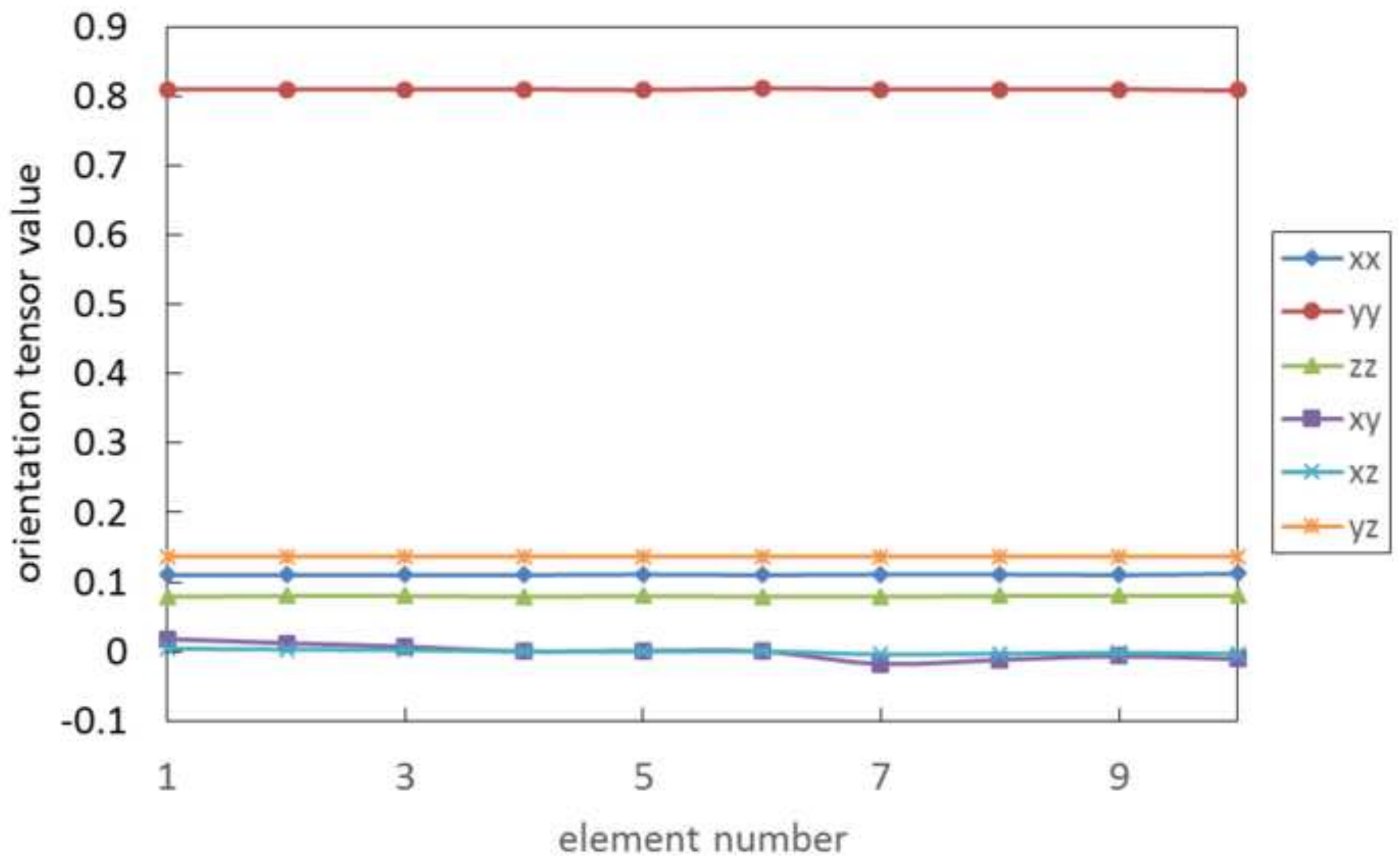




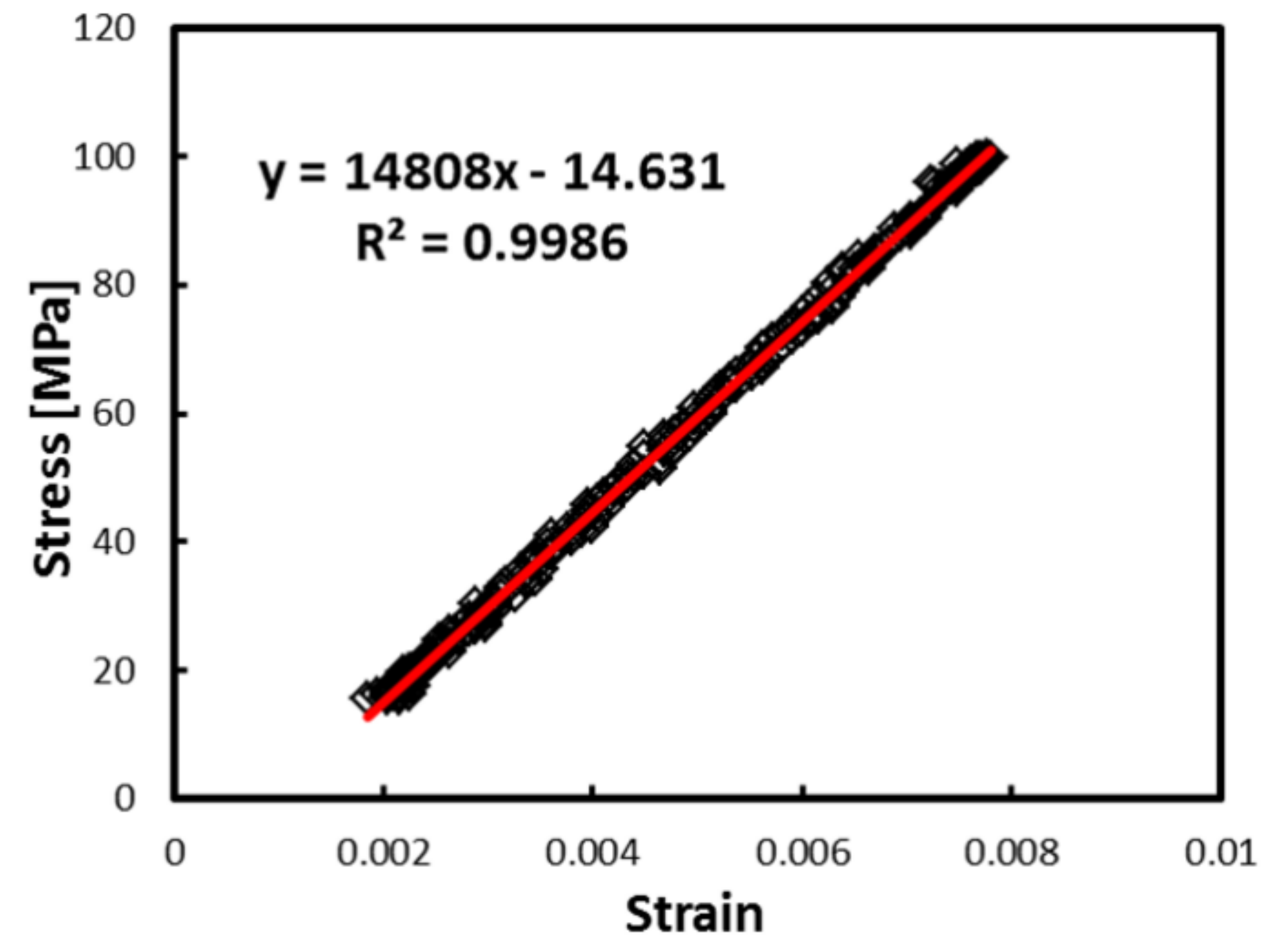




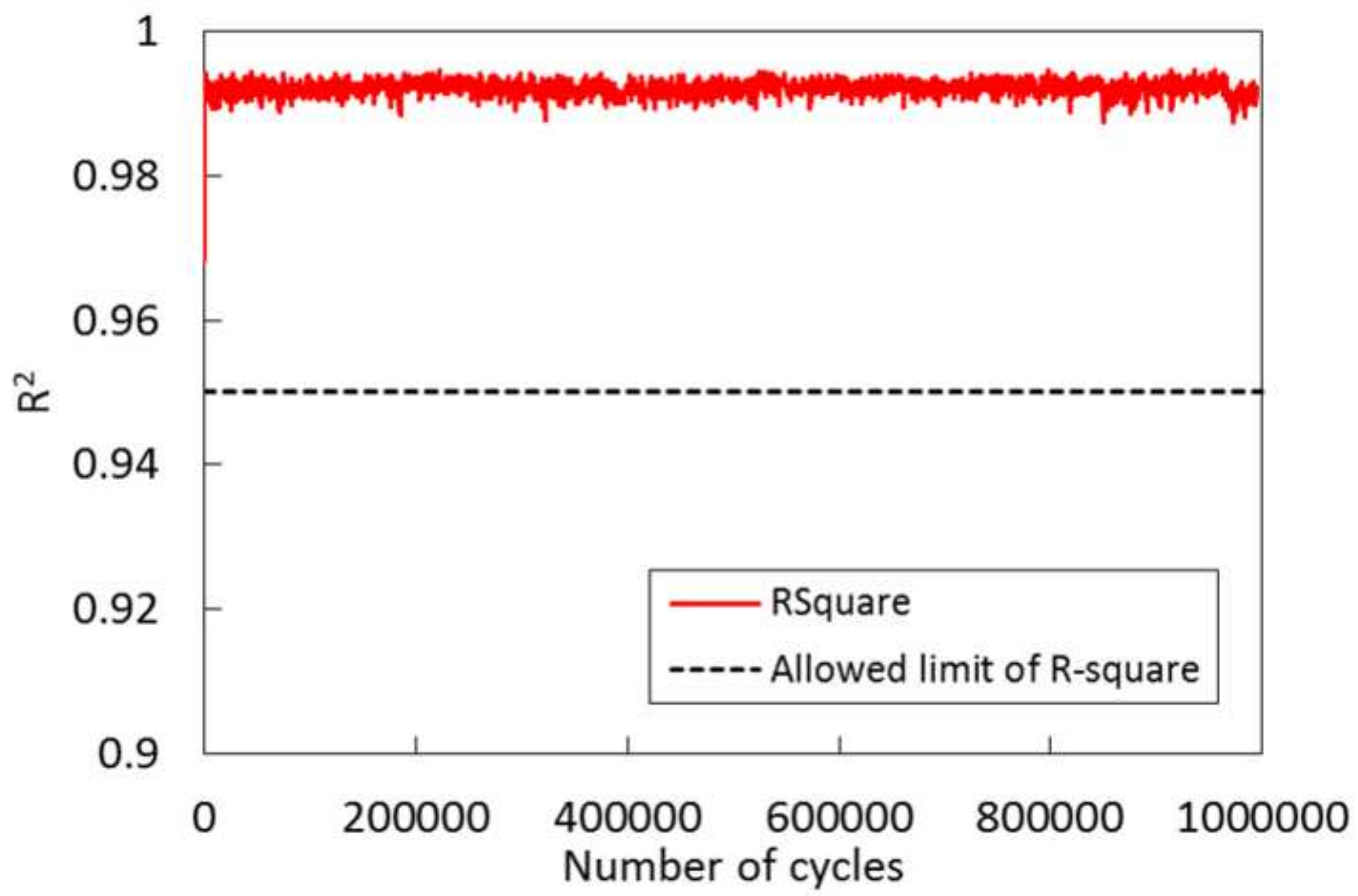




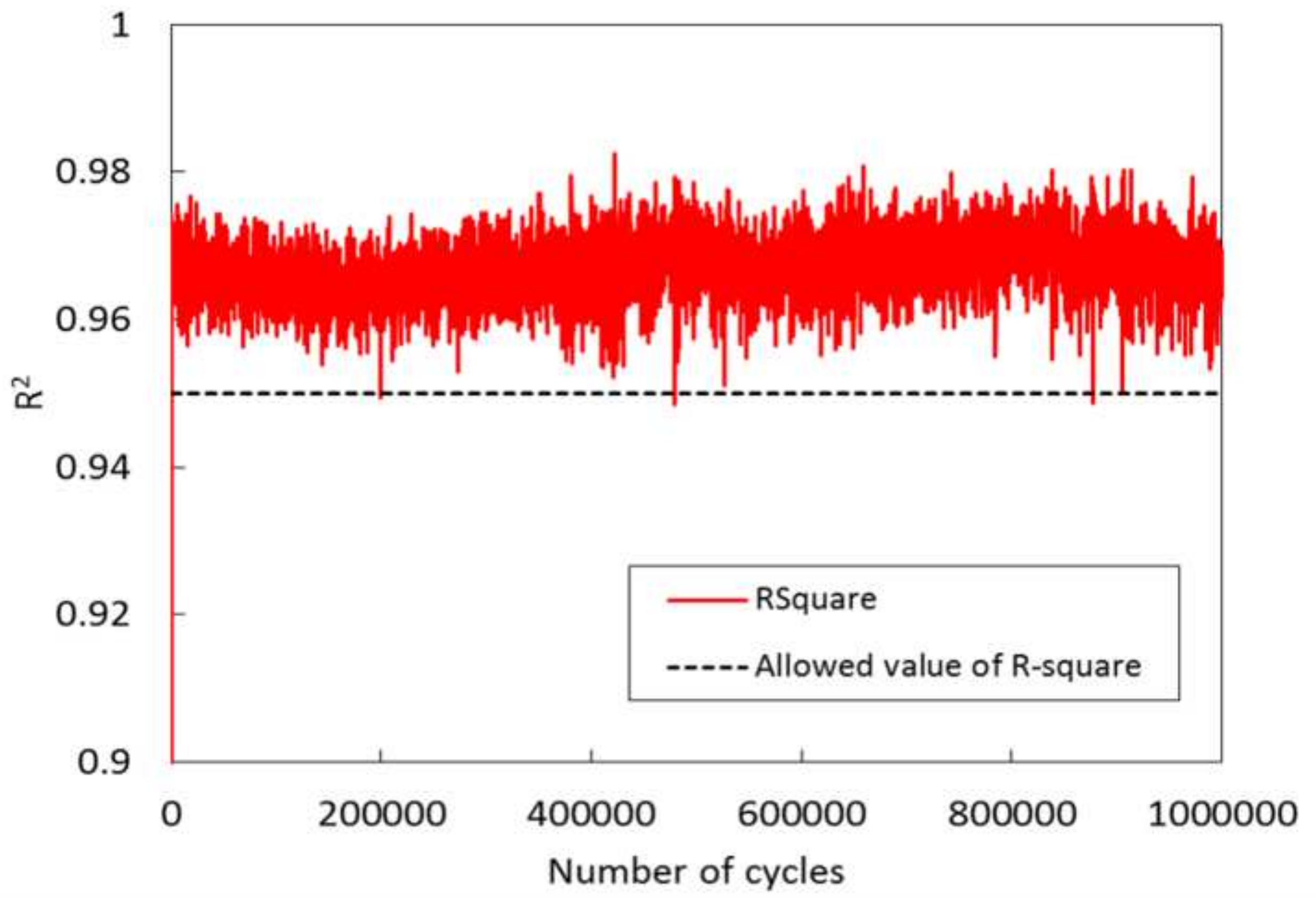


Click here to download high resolution image

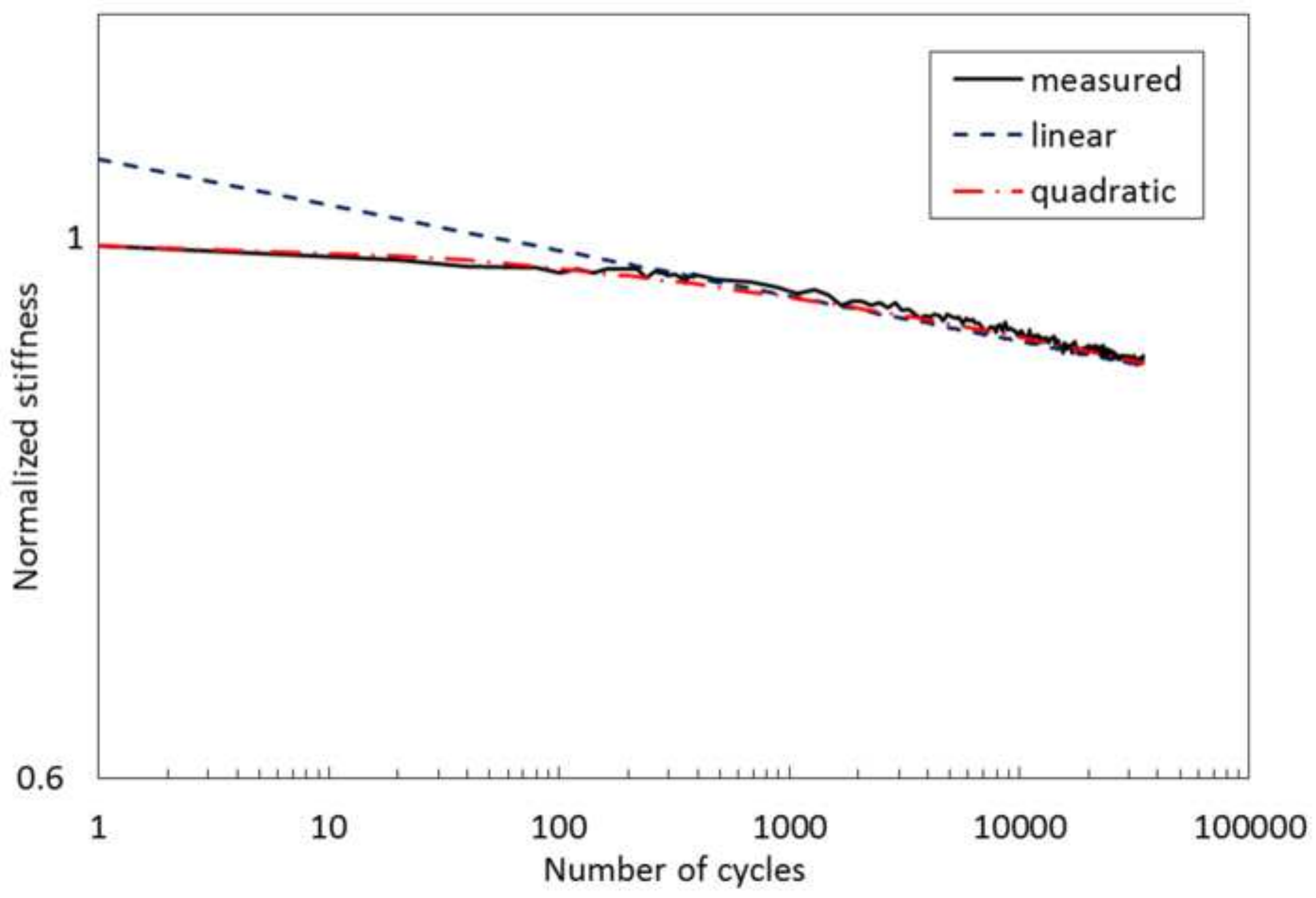




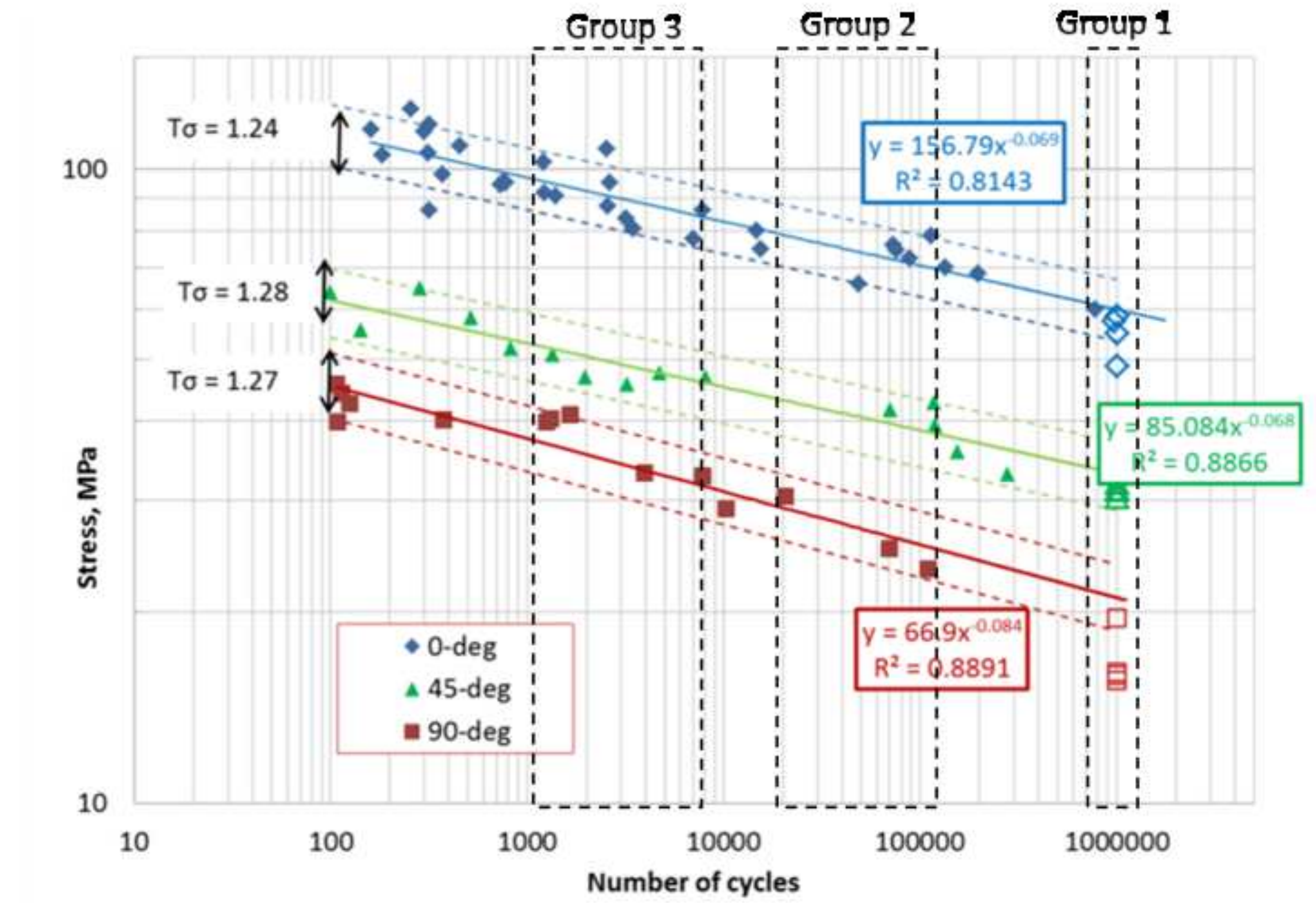

(1)

(1)

(n) 


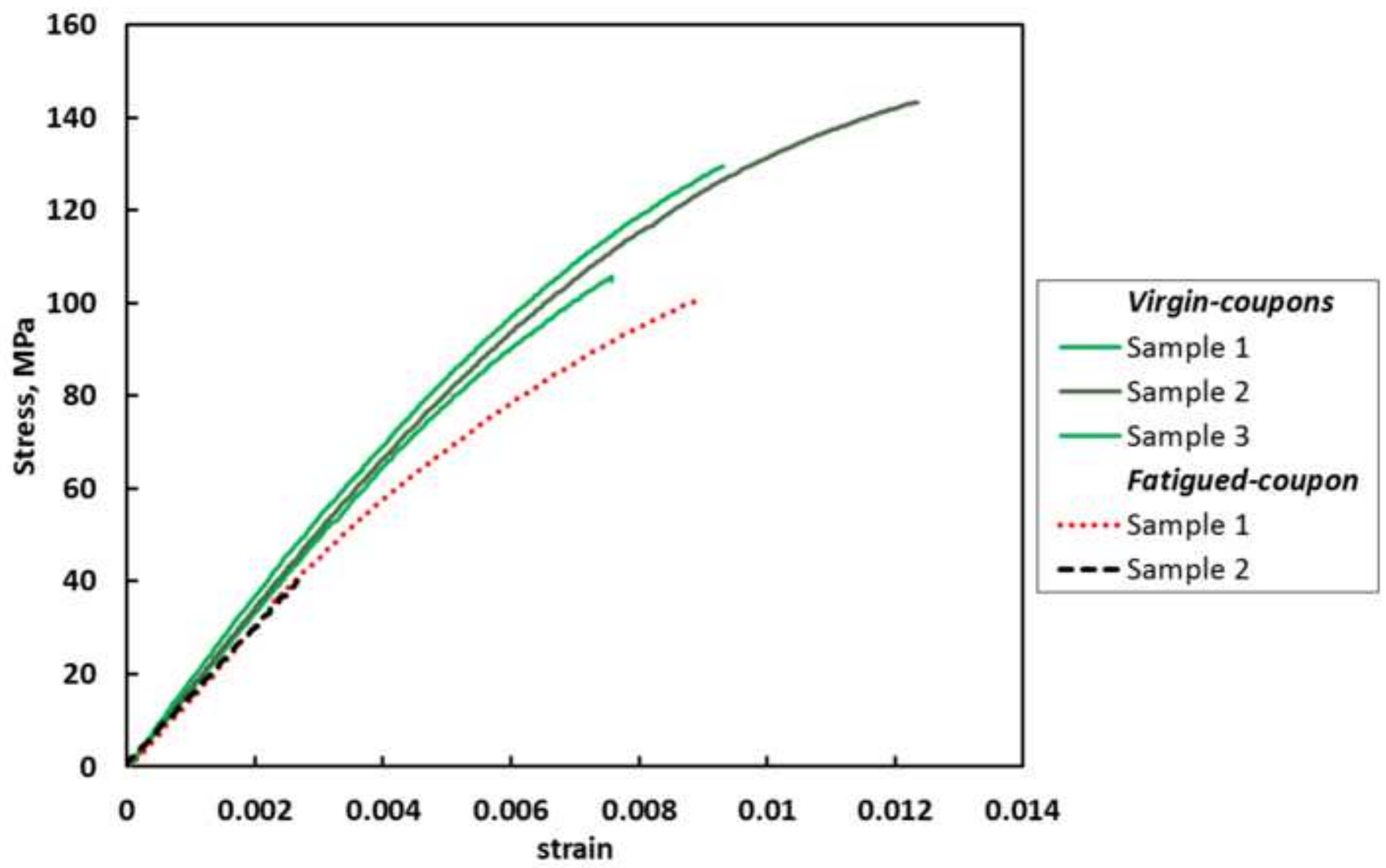




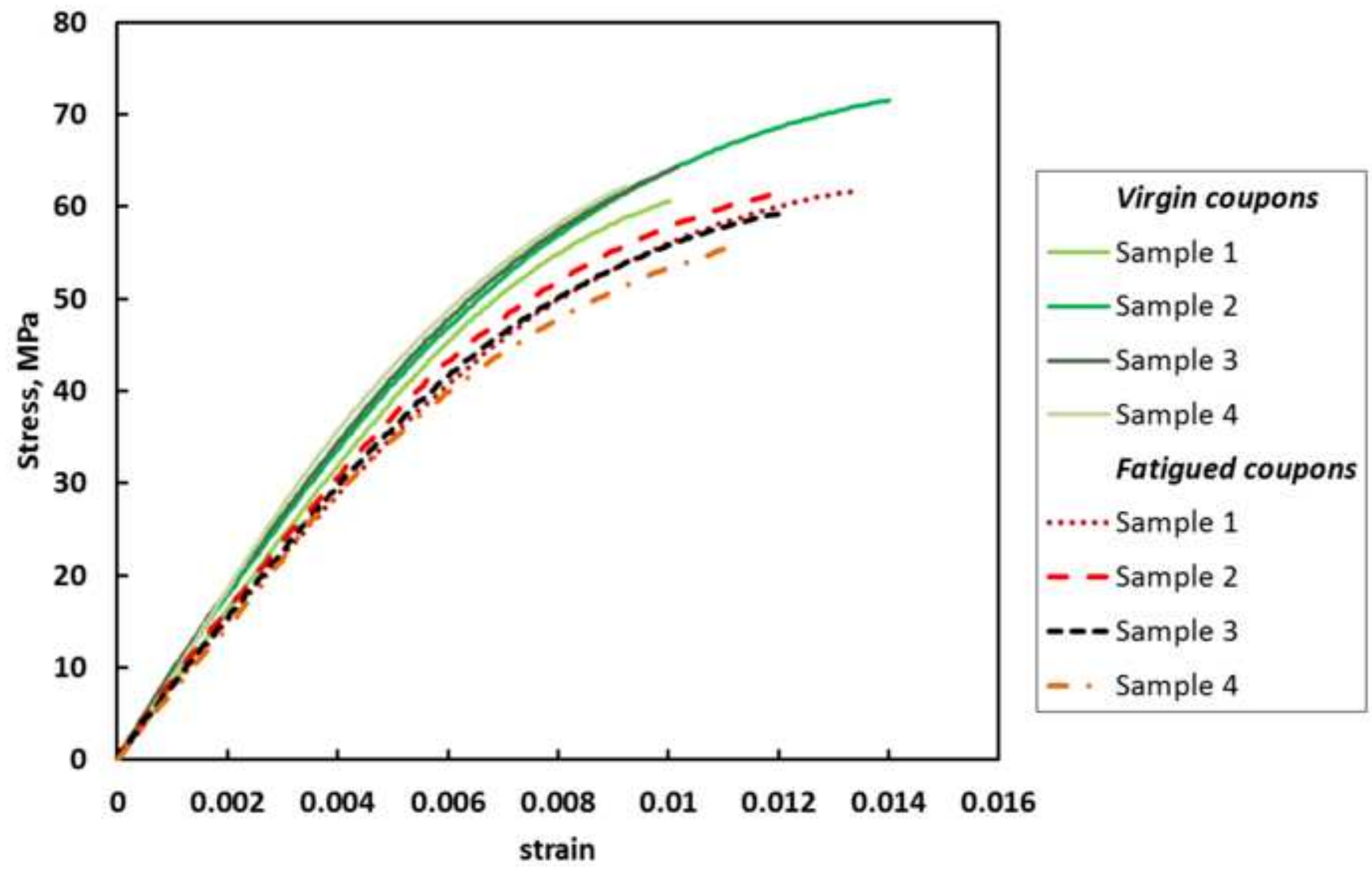


Click here to download high resolution image

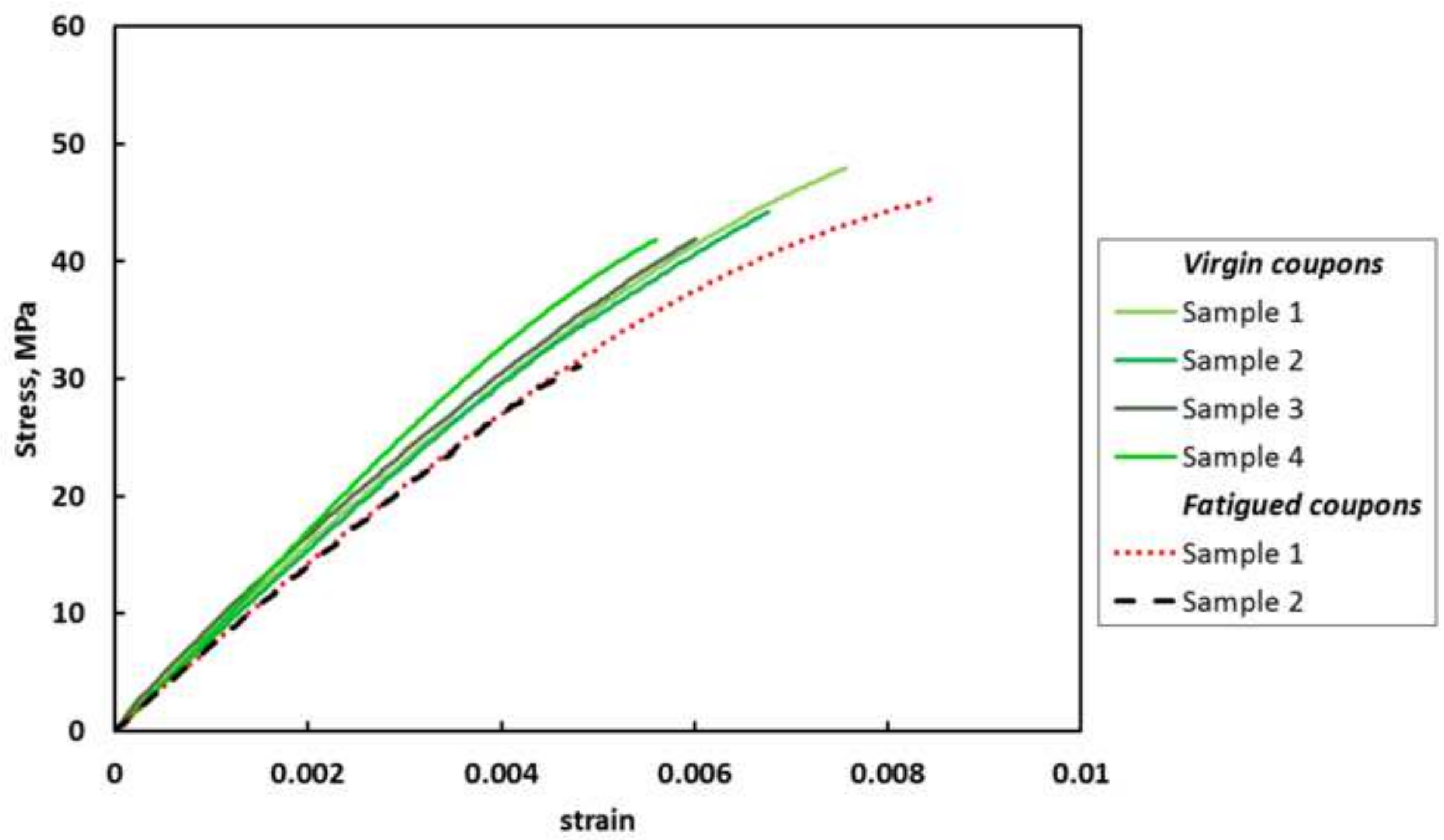




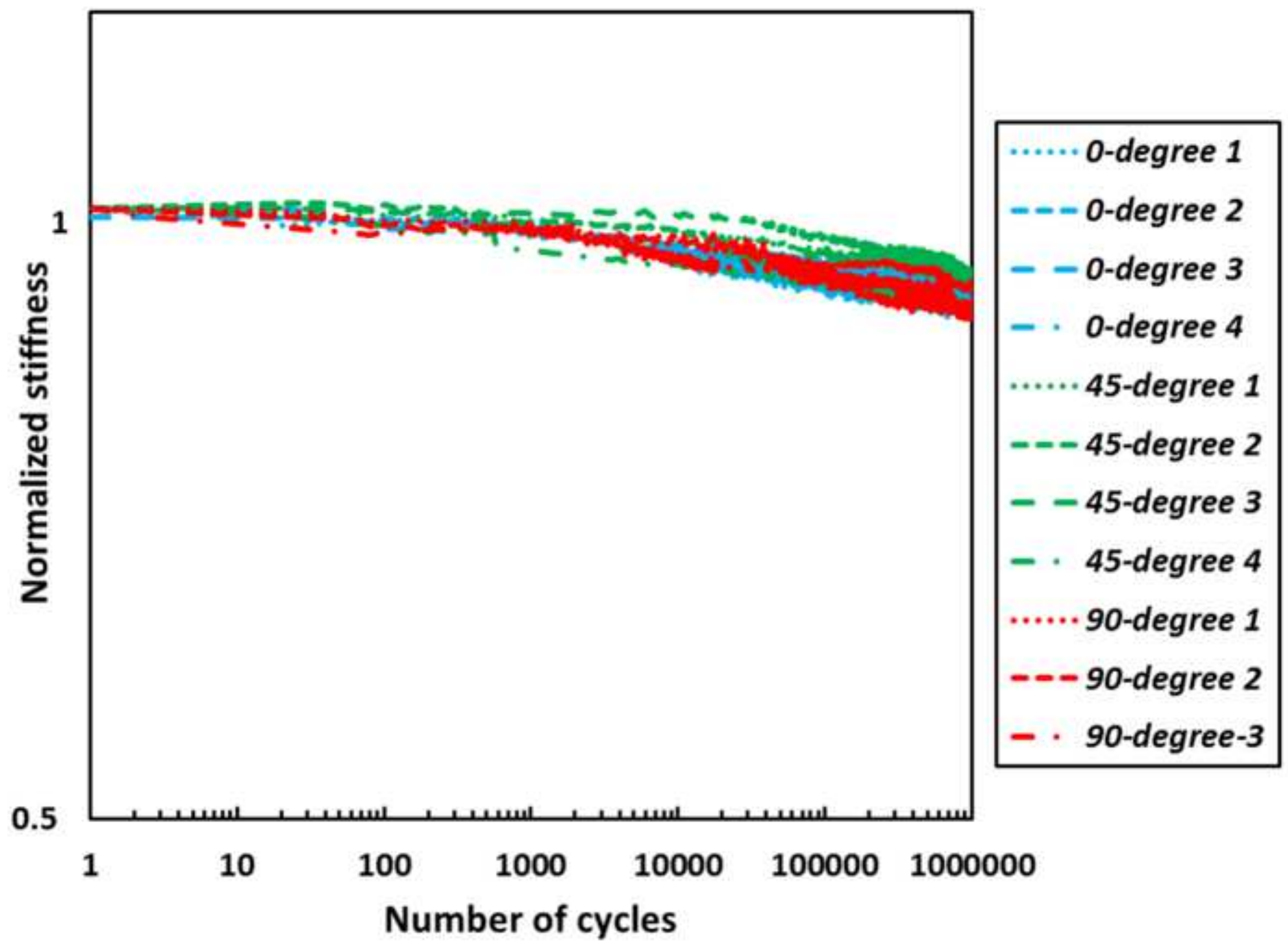




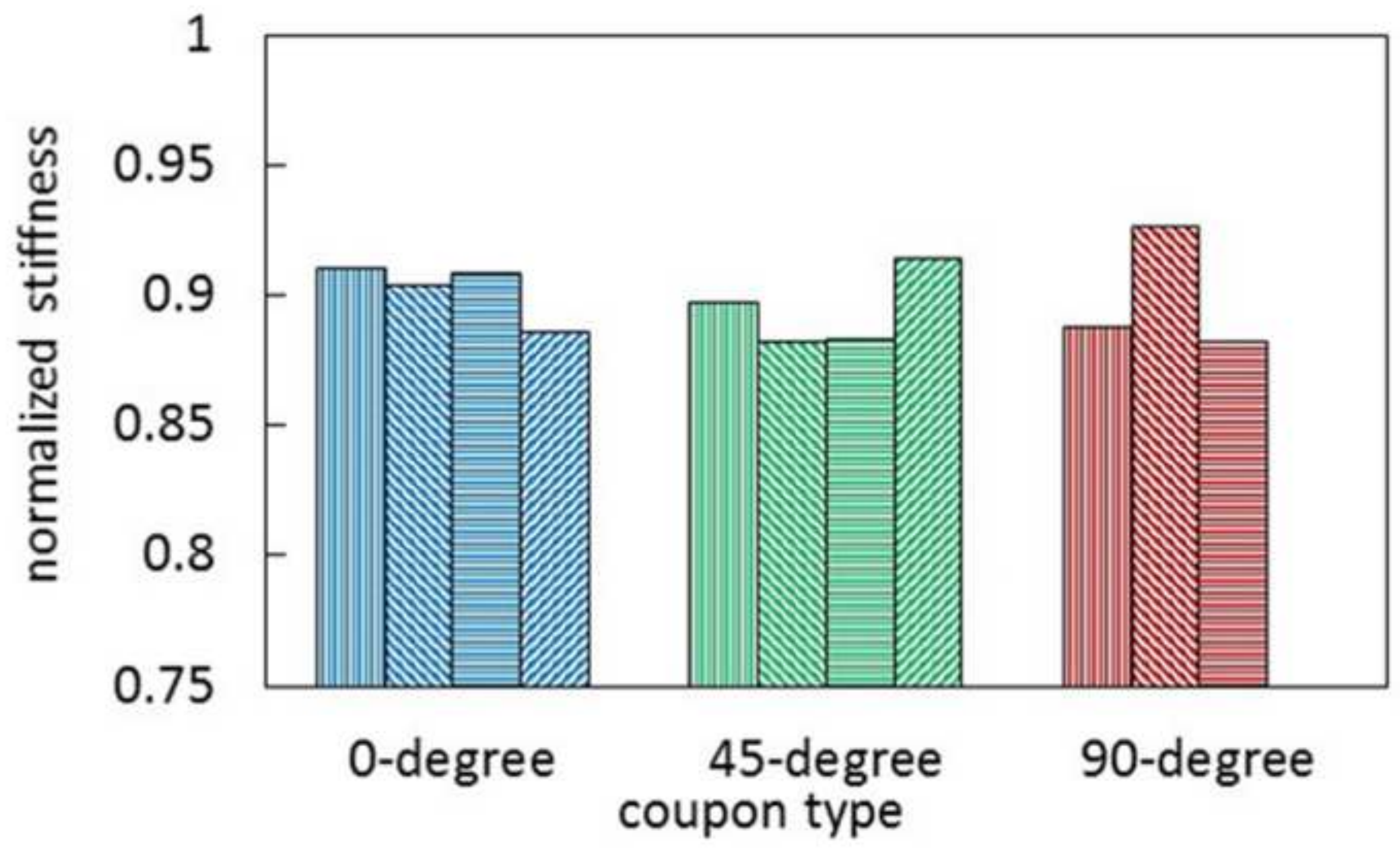




\section{gure 10a}

Click here to download high resolution image

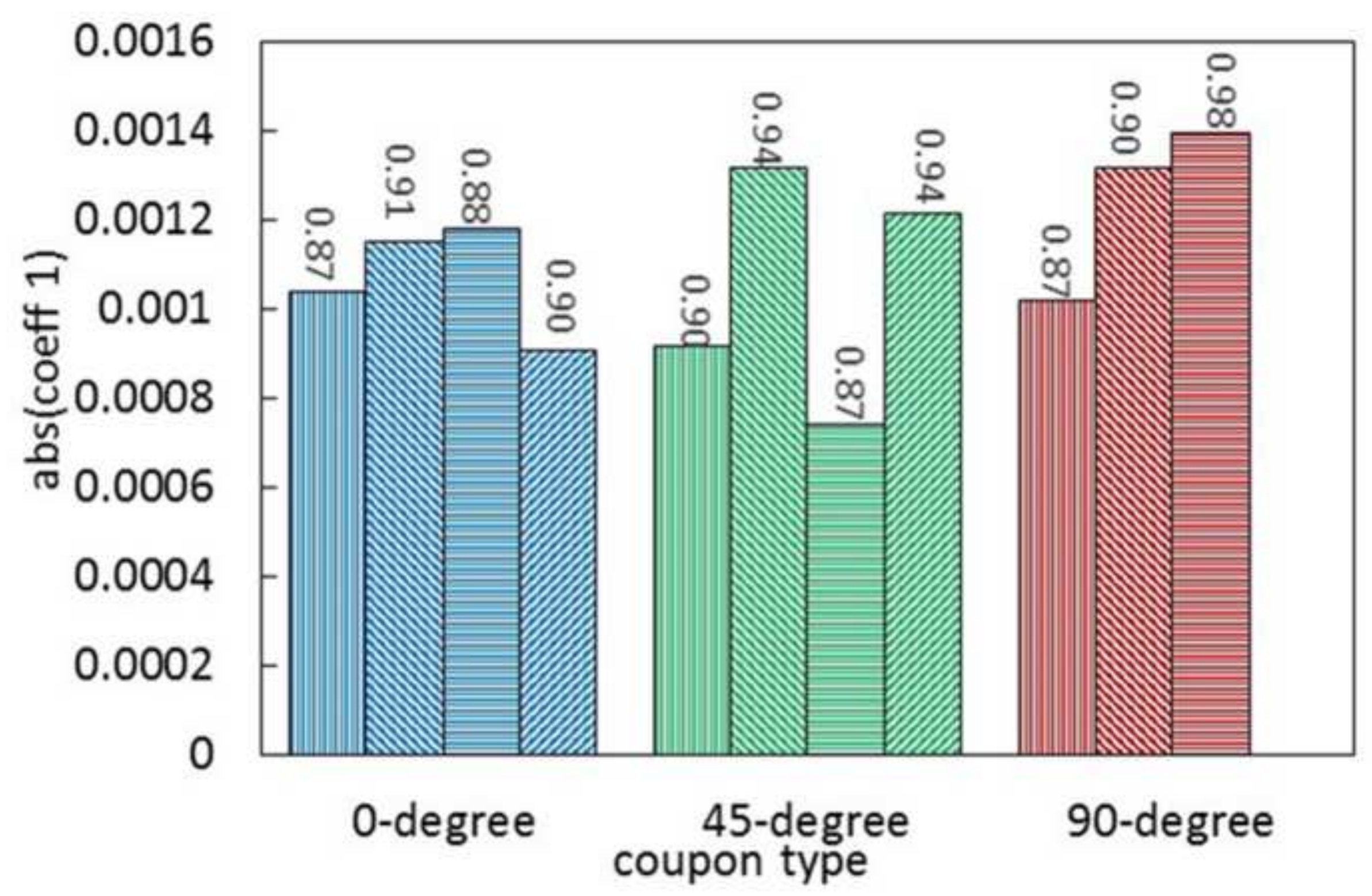



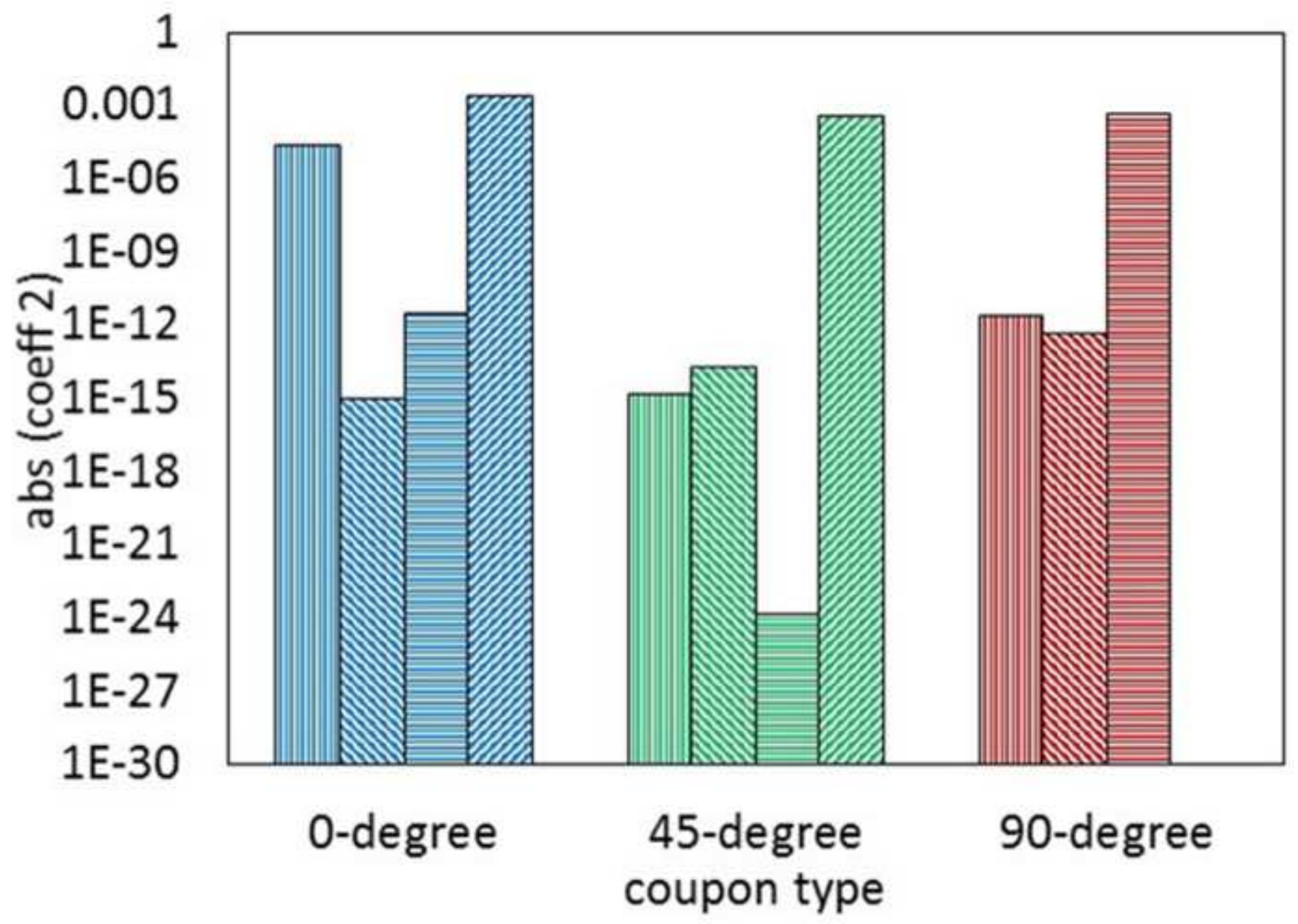


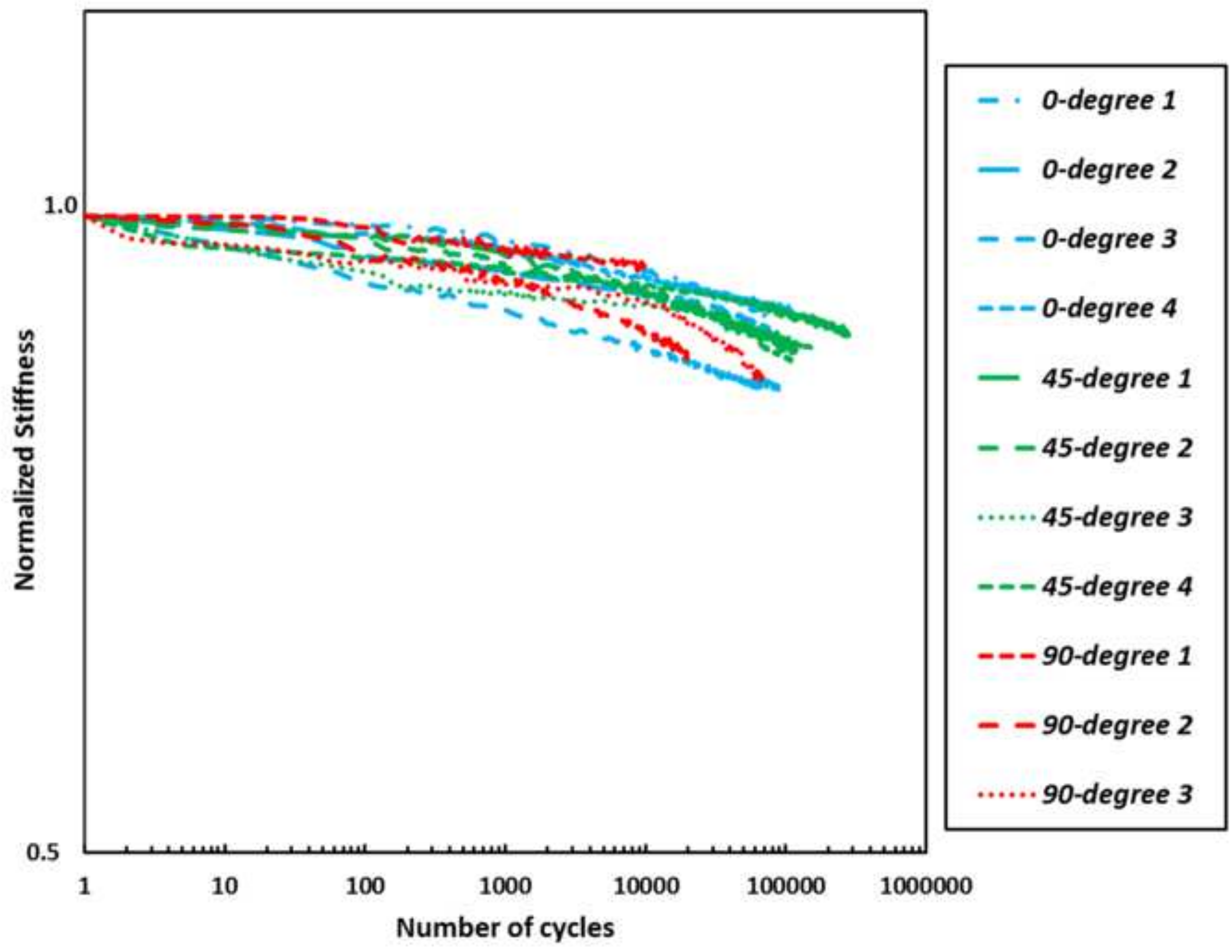




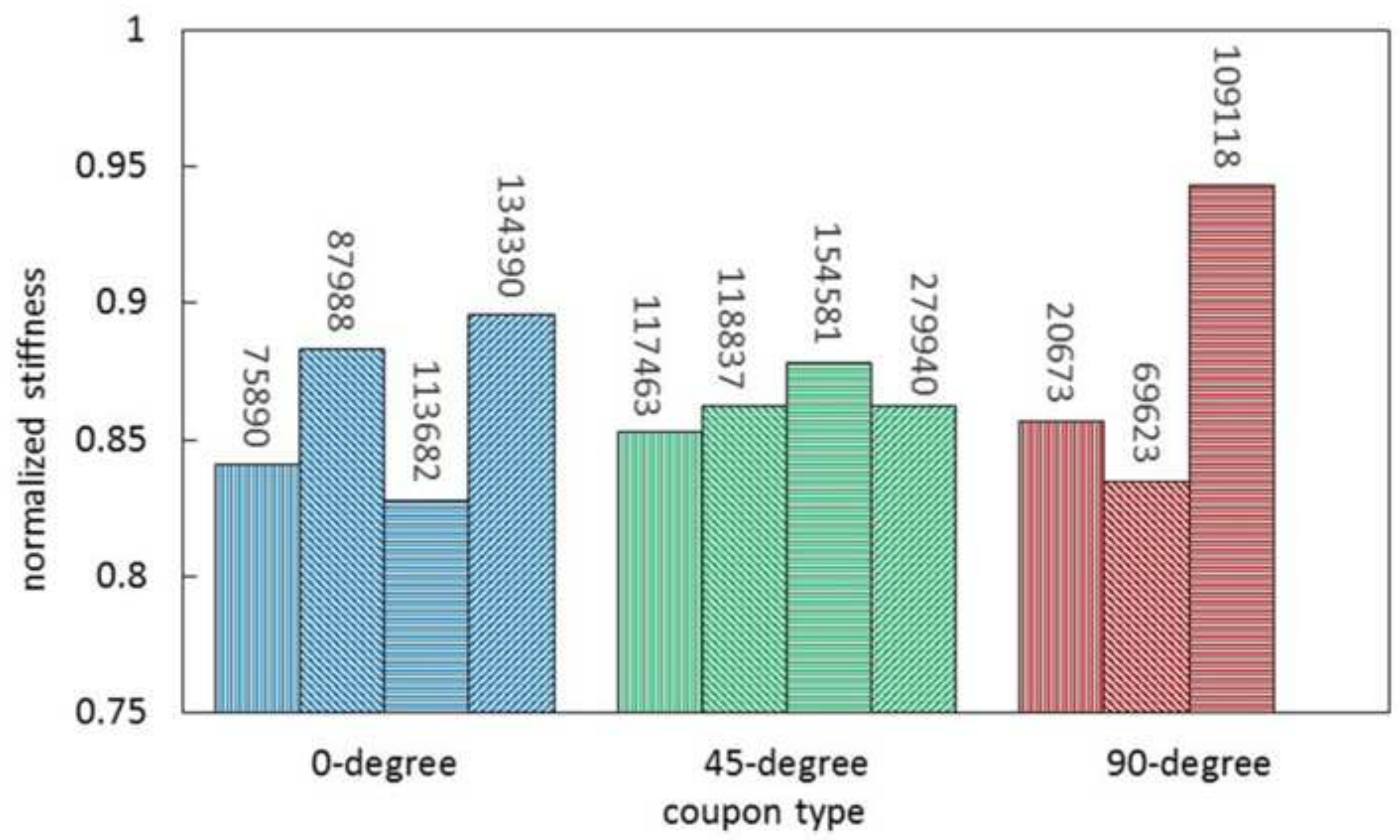




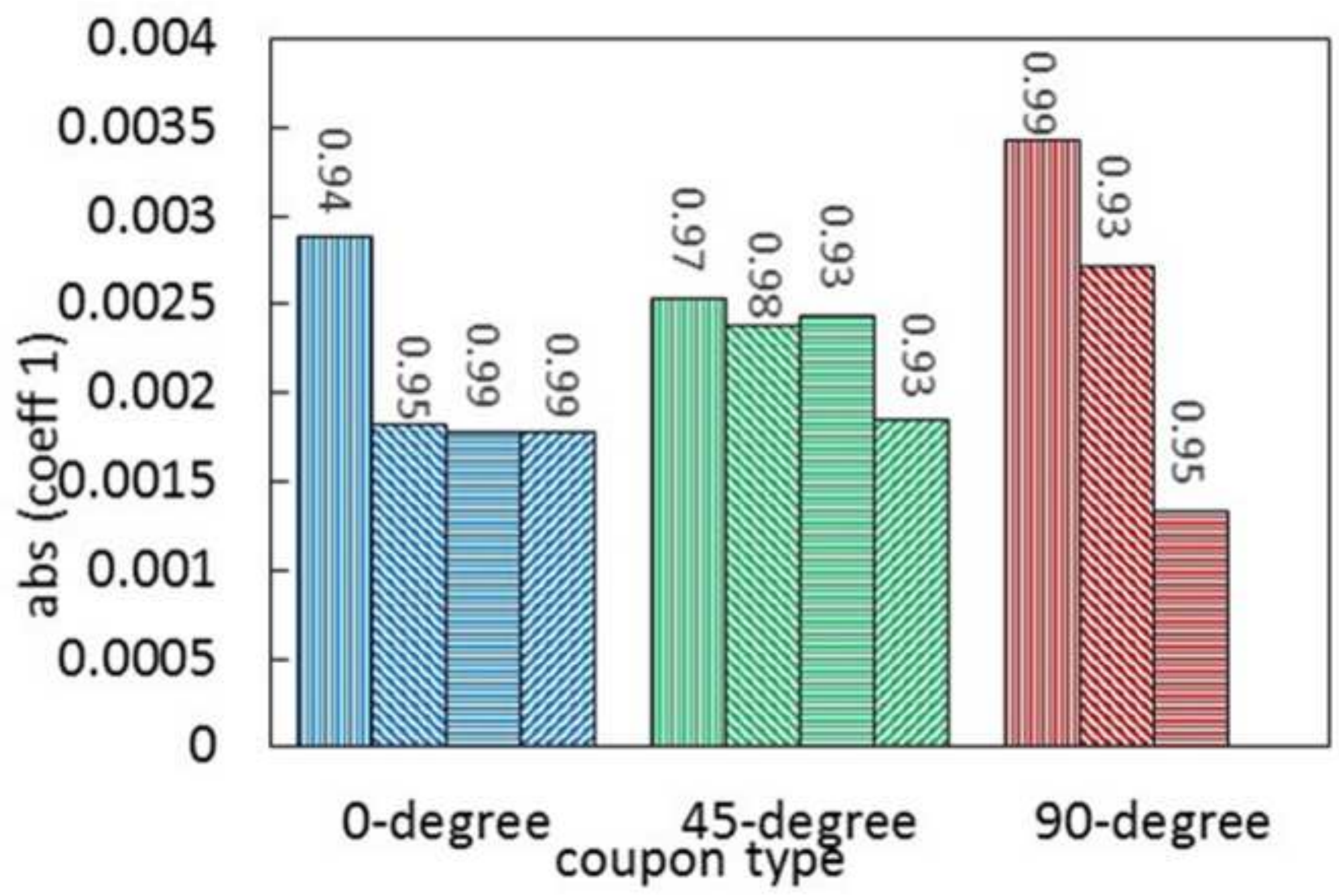




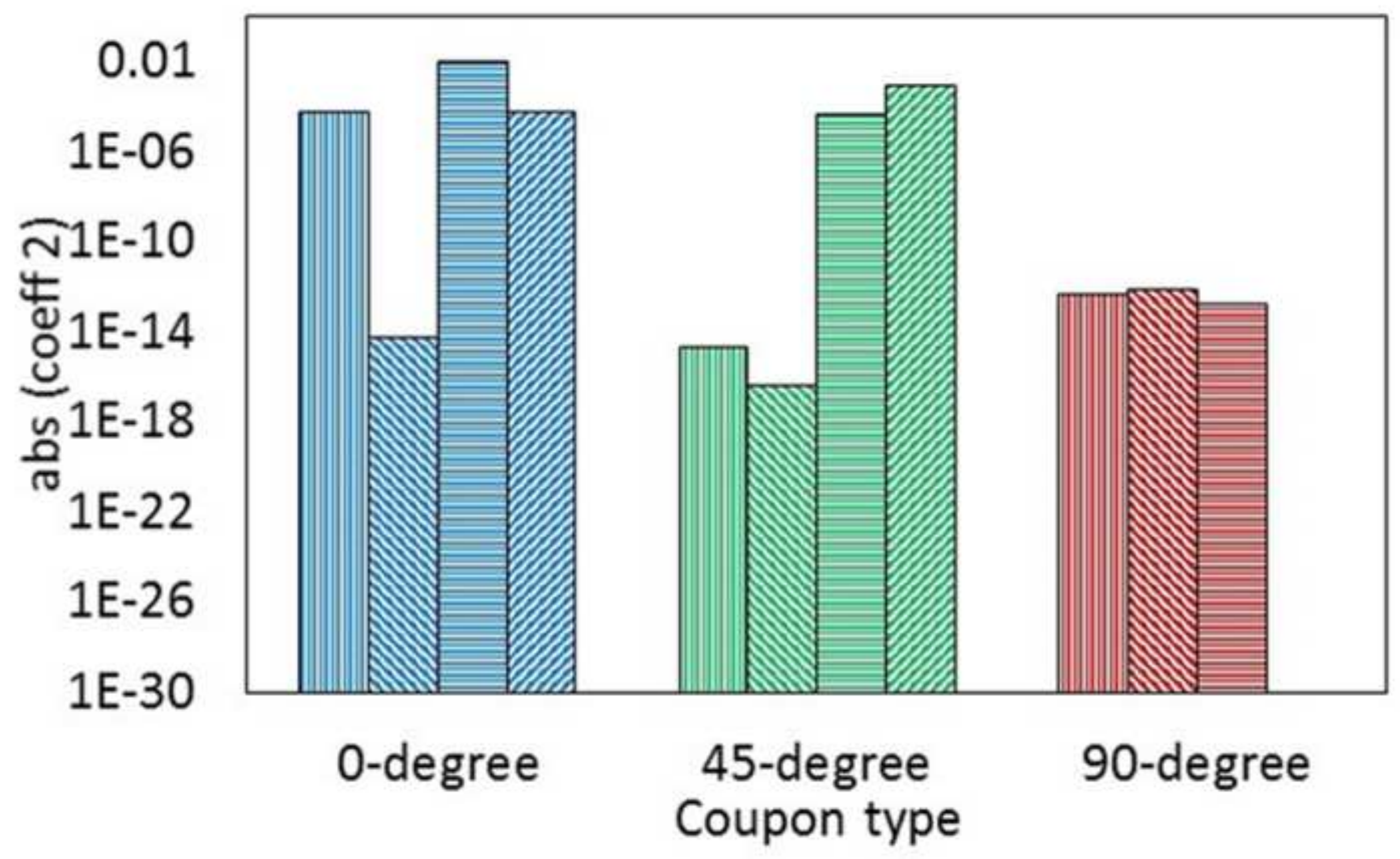




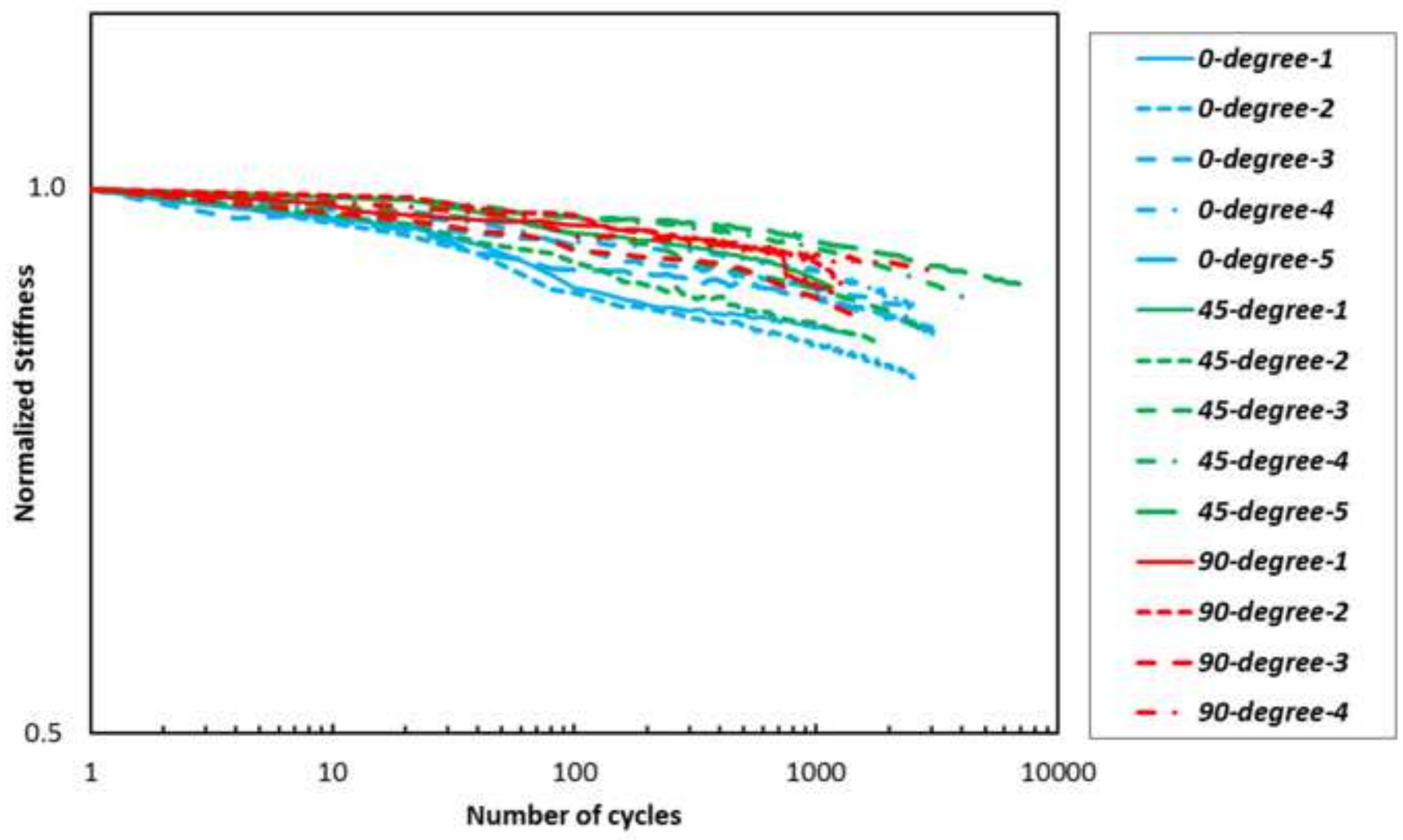




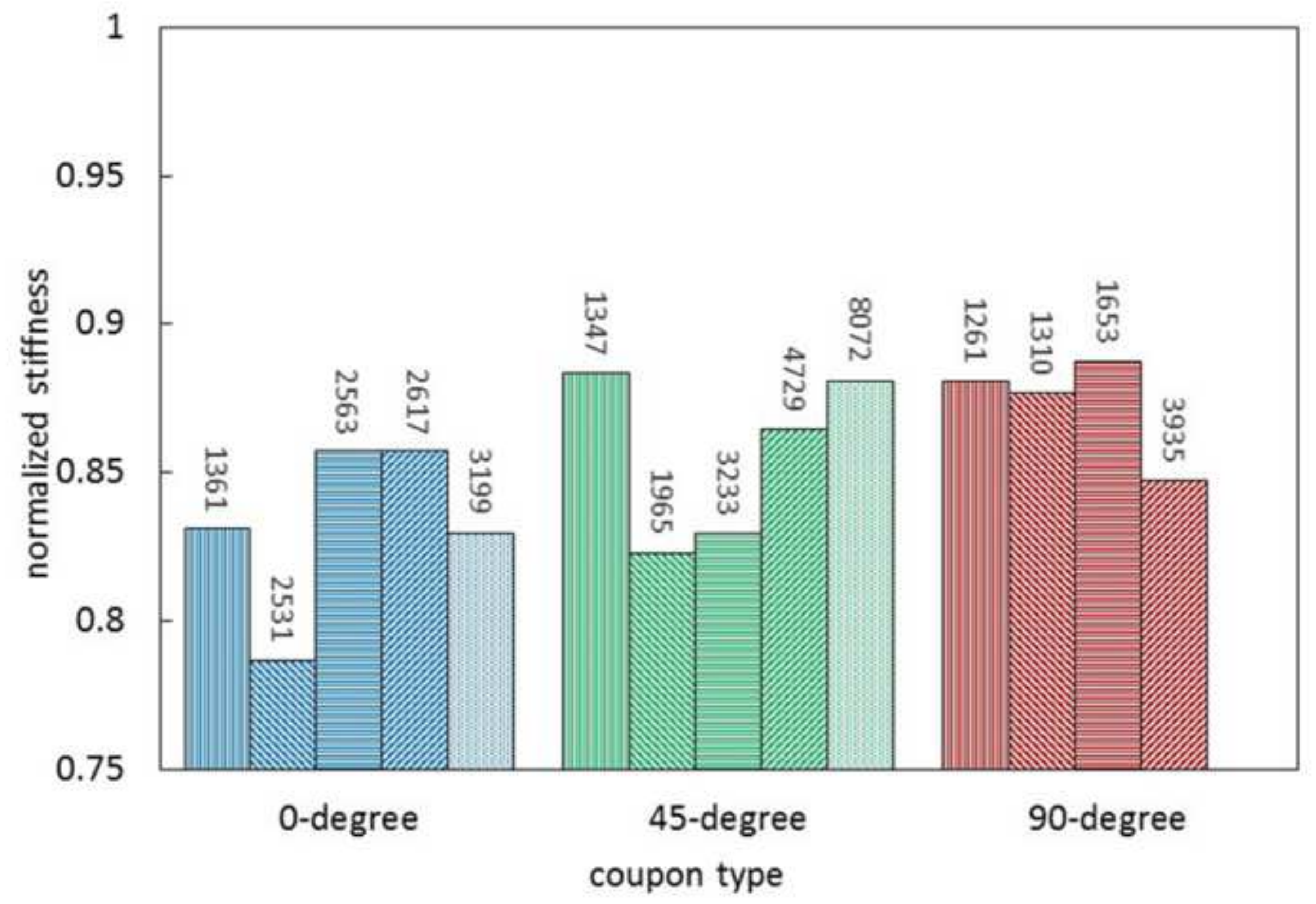




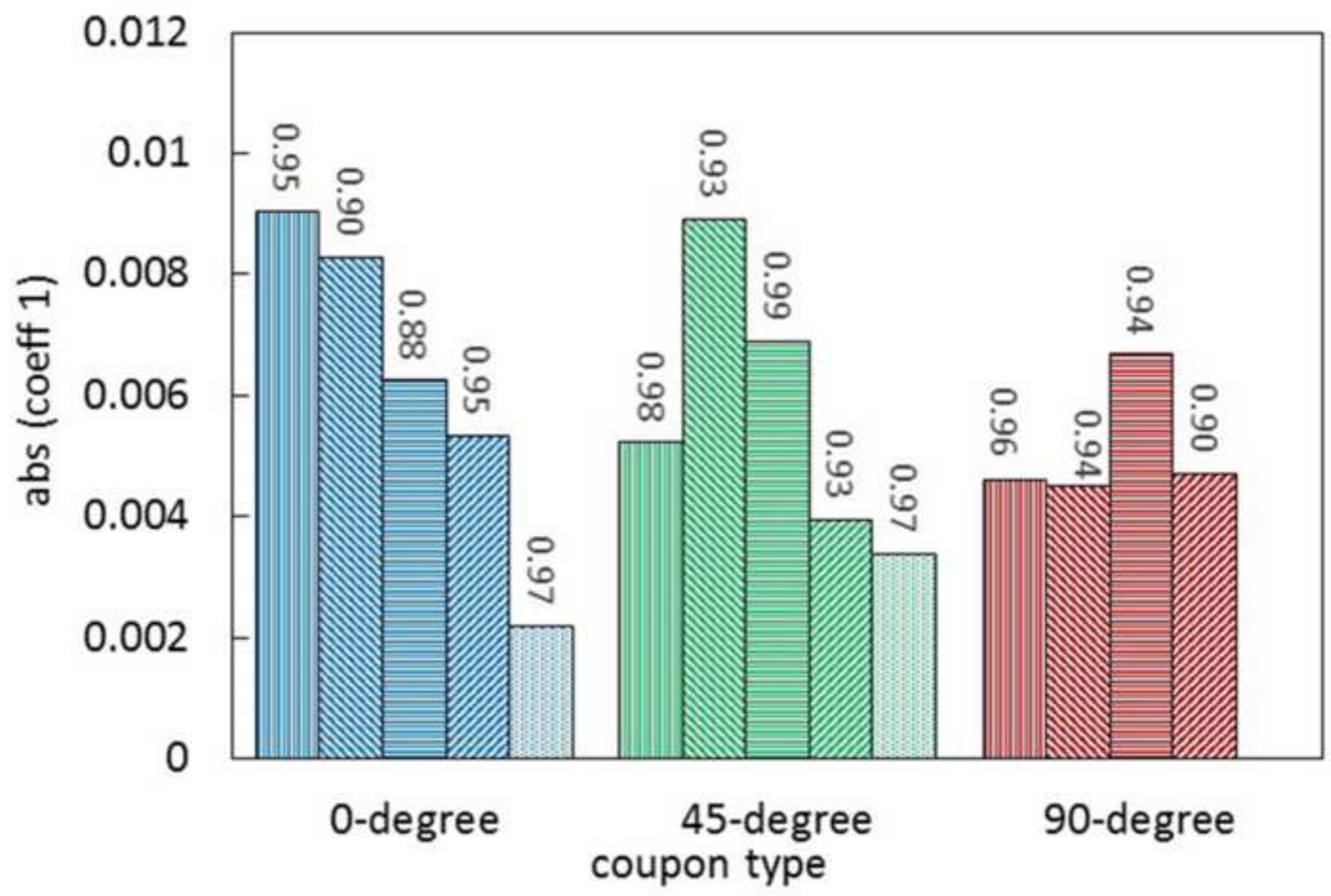




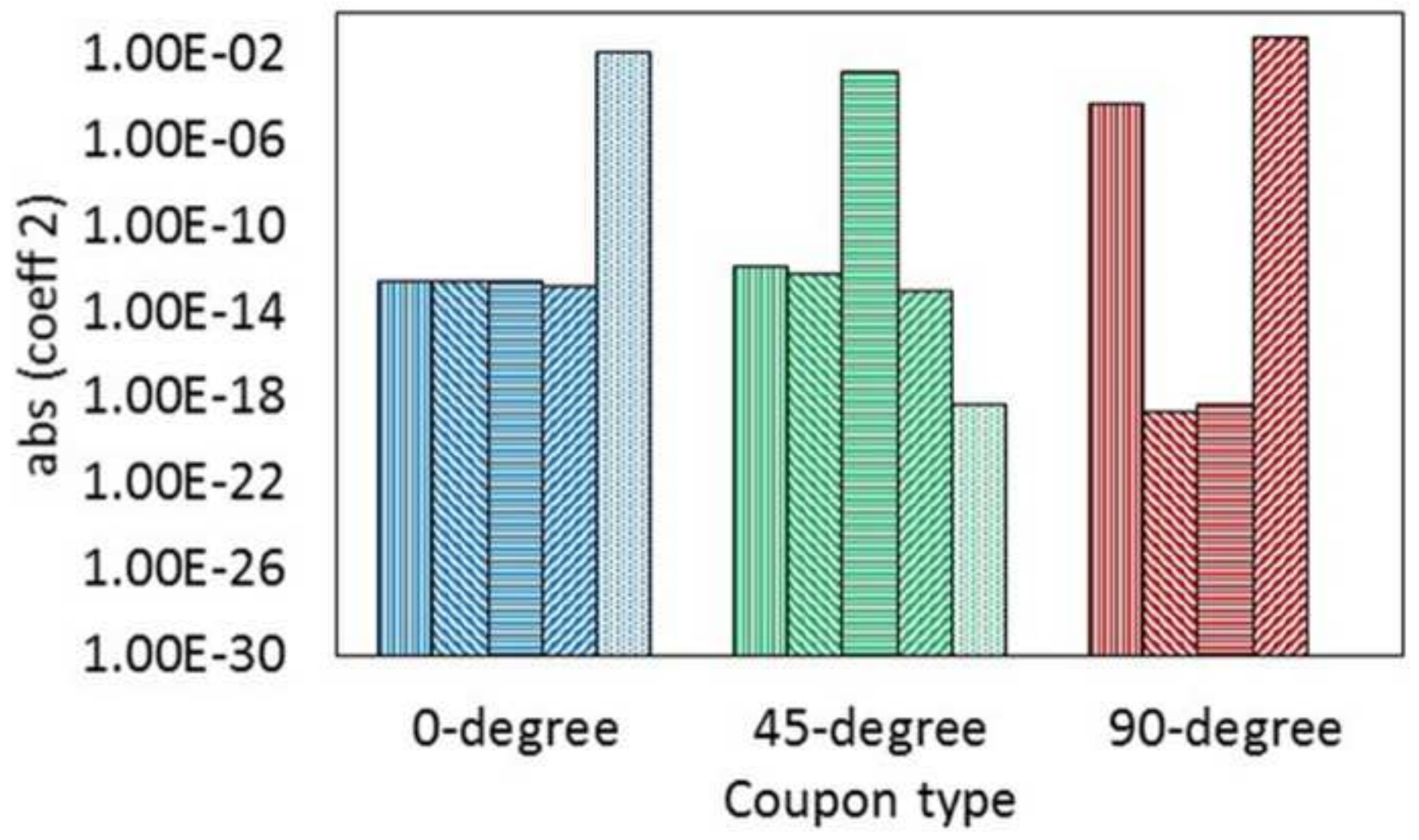

